\title{
MYCL promotes iPSC-like colony formation via MYC Box 0 and 2 domains
}

\section{$\operatorname{AUTHOR}(\mathrm{S})$ :}

Akifuji, Chiaki; Iwasaki, Mio; Kawahara, Yuka; Sakurai, Chiho; Cheng, Yu-Shen; Imai, Takahiko; Nakagawa, Masato

\section{CITATION:}

Akifuji, Chiaki ...[et al]. MYCL promotes iPSC-like colony formation via MYC Box 0 and 2 domains. Scientific Reports 2021, 11: 24254.

\section{ISSUE DATE:}

2021

URL:

http://hdl.handle.net/2433/266645

\section{RIGHT:}

(c) The Author(s) 2021; This article is licensed under a Creative Commons Attribution 4.0 International License, which permits use, sharing, adaptation, distribution and reproduction in any medium or format, as long as you give appropriate credit to the original author(s) and the source, provide a link to the Creative Commons licence, and indicate if changes were made. The images or other third party material in this article are included in the article's Creative Commons licence, unless indicated otherwise in a credit line to the material. If material is not included in the article's Creative Commons licence and your intended use is not permitted by statutory regulation or exceeds the permitted use, you will need to obtain permission directly from the copyright holder. 


\title{
scientific reports
}

Check for updates

\section{OPEN MYCL promotes iPSC-like colony formation via MYC Box 0 and 2 domains}

\author{
Chiaki Akifuji, Mio Iwasaki, Yuka Kawahara, Chiho Sakurai, Yu-Shen Cheng, Takahiko Imai \& \\ Masato Nakagawa
}

Human induced pluripotent stem cells (hiPSCs) can differentiate into cells of the three germ layers and are promising cell sources for regenerative medicine therapies. However, current protocols generate hiPSCs with low efficiency, and the generated iPSCs have variable differentiation capacity among different clones. Our previous study reported that MYC proteins (c-MYC and MYCL) are essential for reprogramming and germline transmission but that MYCL can generate hiPSC colonies more efficiently than c-MYC. The molecular underpinnings for the different reprogramming efficiencies between C-MYC and MYCL, however, are unknown. In this study, we found that MYC Box 0 (MB0) and MB2, two functional domains conserved in the MYC protein family, contribute to the phenotypic differences and promote hiPSC generation in MYCL-induced reprogramming. Proteome analyses suggested that in MYCL-induced reprogramming, cell adhesion-related cytoskeletal proteins are regulated by the MB0 domain, while the MB2 domain regulates RNA processes. These findings provide a molecular explanation for why MYCL has higher reprogramming efficiency than c-MYC.

Human induced pluripotent stem cells (hiPSCs) are generated from somatic cells and can differentiate into cells of all three germ layers ${ }^{1,2}$. They are functionally identical to human embryonic stem cells (hESCs) but do not require the destruction of the embryo, which has made them attractive sources for regenerative medicine ${ }^{3}$. The original reprogramming was induced by four factors, OCT3/4, SOX2, KLF4, and c-MYC (OSKM). Since then, several new methods have been developed to improve the yield and quality of iPSCs, but the cost remains high and the production remains technically difficult ${ }^{4,5}$. Further complicating the application of hiPSCs is the wide variability in the differentiation capacity of different hiPSC clones $^{6}$.

We have shown that excluding c-MYC from the reprogramming factors significantly lowers the reprogramming and differentiation efficiencies of the resulting iPSCs ${ }^{7}$. The MYC family consists of the oncogenes c-MYC, MYCN, and MYCL in humans ${ }^{8}$. c-MYC was the first MYC gene discovered in human and has been a topic of cancer research ever since ${ }^{9}$. Tumorigenesis depends on high transformation activity derived from the $\mathrm{N}$-terminus region of c-MYC protein ${ }^{10}$. Consequently, OSKM-based reprogramming may not be appropriate for the clinical application of iPSCs. Many groups have reported reprogramming methods that exclude c-MYC overexpression but at the cost of lower reprogramming efficiency ${ }^{5,7}$. MYCL is about 30 amino acids shorter in the N-terminus region than c-MYC and has lower transformation activity ${ }^{10}$. We found that substituting c-MYC for MYCL in reprogramming can increase the number of iPSC colonies and maintain the ability to differentiate into the cells of three germ layers ${ }^{7}$. Furthermore, fewer chimeric mice died by tumorigenesis after the transplantation of MYCL-iPSCs, whereas the transplantation of c-MYC-iPSCs caused lethal tumorigenesis in more than $50 \%$ of mice during two years of observation. Despite these observations, little is known about the molecular function of MYCL and the different mechanisms between c-MYC and MYCL to promote reprogramming.

MYC proteins have six MYC Box (MB) domains: MB0, 1, 2, 3a, 3b, and 4 in the N-terminus and a basic helixloop-helix leucine zipper (bHLHLZ) in the C-terminus ${ }^{11}$, but MYCL does not have MB3a. The C-terminus of c-MYC and MYCL is essential in reprogramming due to its binding with MAX protein, allowing MYC to access the $\mathrm{DNA}^{7,12}$. The $\mathrm{N}$-terminus is mainly known as a transactivation domain (TAD), which regulates the target gene, but its function in reprogramming is less clear ${ }^{13}$. We found that a mutant of c-MYC lacking the N-terminal showed low transformation activity and promoted reprogramming ${ }^{7}$. However, which domain on the $\mathrm{N}$-terminal side is essential for reprogramming and what function it performs were not resolved. In addition, MYC proteins act as transcription factors upon interacting with several binding proteins ${ }^{14}$. Although MYCL-binding proteins are important for MYCL function, there are no reports about MYCL-binding proteins during reprogramming.

Department of Life Science Frontiers, Center for iPS Cell Research and Application (CiRA), Kyoto University, Kyoto 606-8507, Japan. ${ }^{\square}$ email: nakagawa@cira.kyoto-u.ac.jp 
A

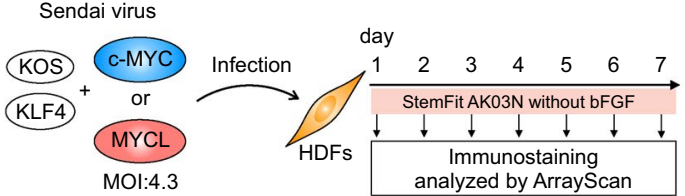

B

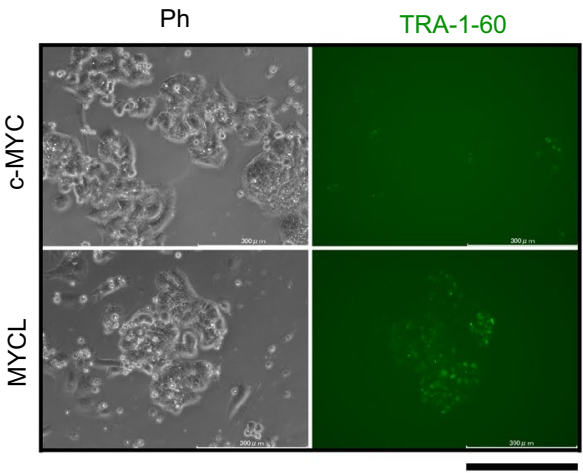

D Episomal plasmid vector

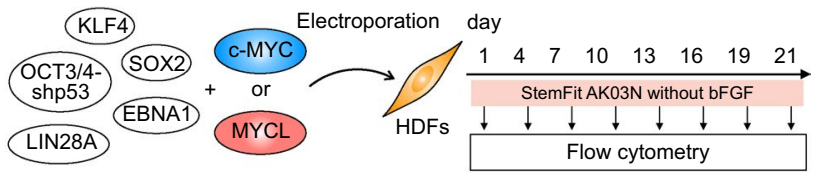

E

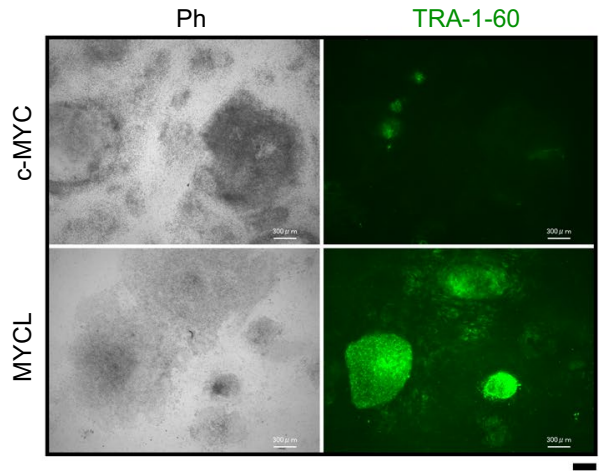

G

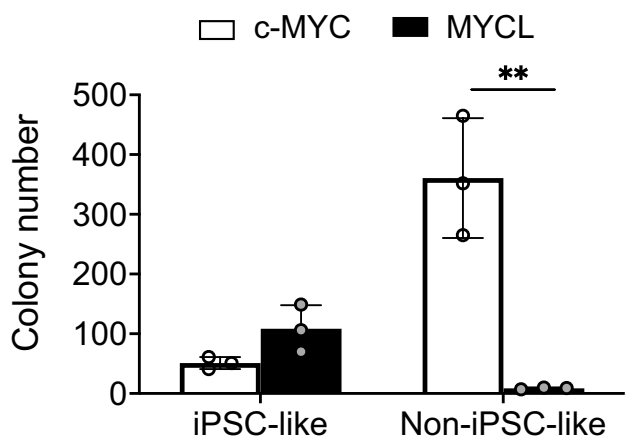

C

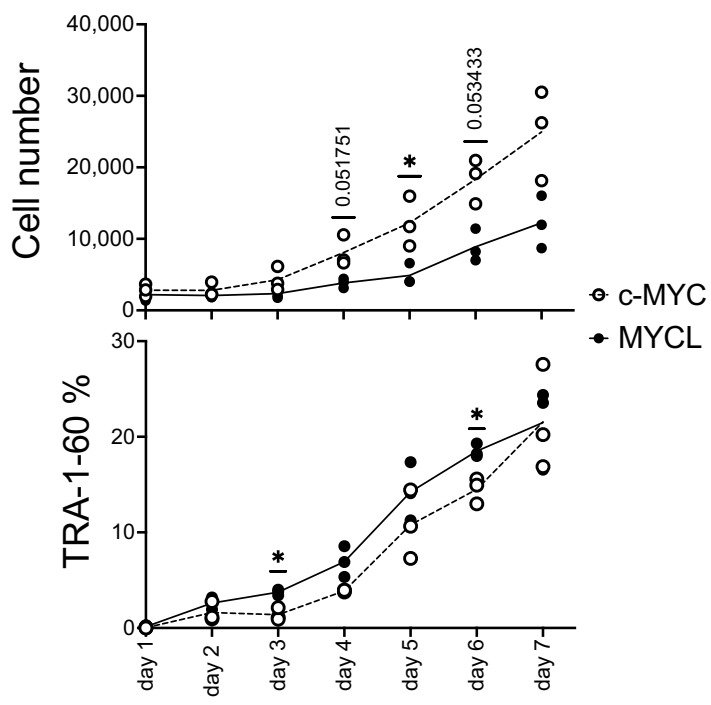

$\mathbf{F}$

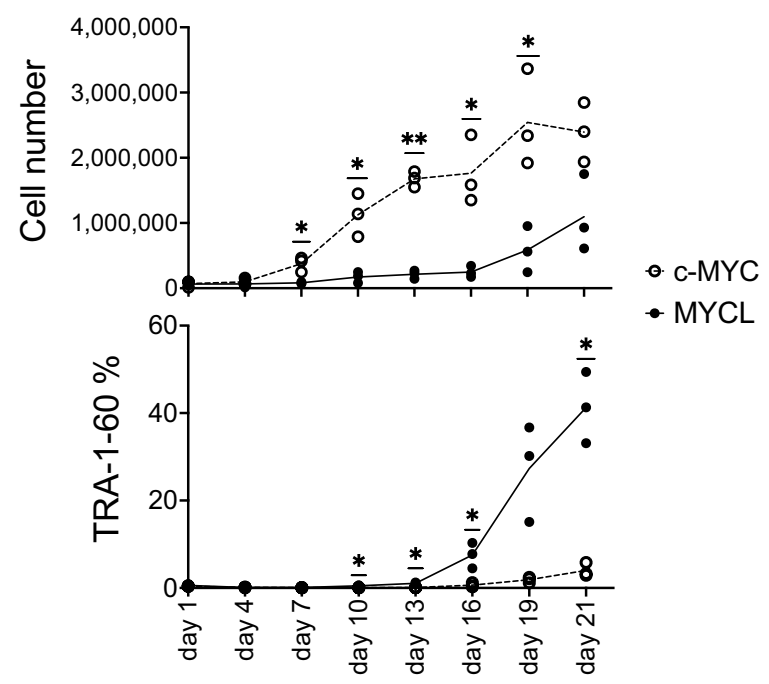

H

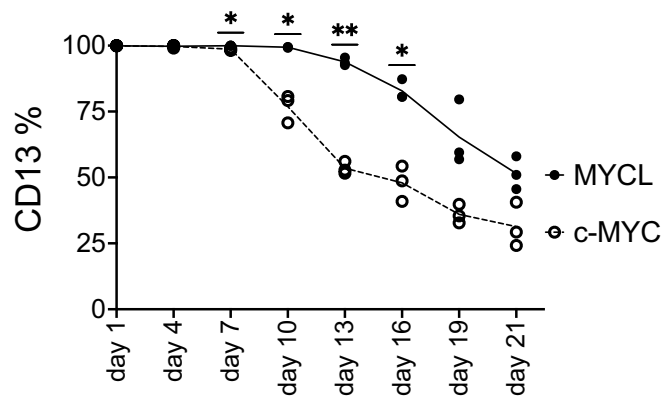


4Figure 1. MYCL promotes reprogramming more efficiently than c-MYC. (A) Schematic representation of HDF reprogramming with Sendai virus (SeV). HDFs were transduced with SeV carrying KLF4-OCT3/4-SOX2 (KOS), KLF4 (K), and c-MYC or MYCL on day 0 . We used an MOI (multiplicity of infection) of 4.3 for each virus. StemFit AK03N without bFGF was used during the transduction and subsequent induction of iPSC-like colonies. We performed immunostaining of the reprogramming HDFs 1 to 7 days after the transduction and analyzed the results using ArrayScan. (B) Representative immunostaining images of reprogramming HDFs stained by anti-TRA-1-60 antibody (green) and Hoechst (blue) 7 days after the transduction. Scale bar, $300 \mu \mathrm{m}$. $\mathrm{Ph}$, phase contrast. (C) Proliferation and expression of TRA-1-60 (+) cells during reprogramming. HDFs were transduced with $\mathrm{SeV}$, including c-MYC or MYCL, and immunostaining was performed from days 1 to 7 . The number of total cells was counted as Hoechst-positive cells. Mean \pm SD values are shown. $n=3,{ }^{*} p<0.05$ by paired $t$-test. (D) Schematic representation of HDF reprogramming with episomal plasmid vector (EpiP). HDFs were transduced with EpiP carrying SOX2, KLF4, OCT3/4-shp53, LIN28A, EBNA1, and c-MYC or MYCL. StemFit AK03N without bFGF was used during the transfection and subsequent induction of iPSC-like colonies. We performed flow cytometry of the reprogramming HDFs every three days from 1 to 19 days plus day 21 after the transduction. (E) Representative immunostaining images of reprogramming HDFs stained by anti-TRA-1-60 antibody (green) and Hoechst (blue) 21 days after the transduction. Scale bar, $300 \mu \mathrm{m}$. Ph, phase contrast. (F) Proliferation and expression of TRA-1-60 (+) cells during reprogramming were analyzed by flow cytometry. HDFs were transduced with EpiP, including c-MYC or MYCL. Flow cytometry was performed every three days from days 1 to 19 days plus day 21 . Mean \pm SD for $n=3,{ }^{\star} p<0.05$ and ${ }^{\star *} p<0.01$ by paired $t$-test. (G) The number of iPSC-like and non-iPSC-like colonies derived from $1 \times 10^{5} \mathrm{HDF}$ transduced with EpiP including c-MYC or MYCL on day 21. Mean \pm SD values are shown. $n=3,{ }^{* *} p<0.01$ by unpaired $t$-test. (H) Percentage of $\mathrm{CD} 13(+)$ cells during EpiP reprogramming determined by flow cytometry. Mean \pm SD values are shown. $n=3$, ${ }^{\star} p<0.05$ and ${ }^{\star *} p<0.01$ by paired $t$-test.

In this study, using domain deletion mutants of MYC proteins, we found that the MB0 and MB2 domains promote iPSC-like colonies and that the MB0 domain is functionally different between c-MYC and MYCL. In c-MYC, it induced non-iPSC-like colonies by increasing nucleic proteins related to transcription, but in MYCL, the MB0 domain induced iPSC-like colonies by increasing the expression of cell adhesion-related proteins. We also found that deletion of the MB2 domain in MYC proteins prevented colony formation and that MYCL could interact with RNA-binding proteins (RBPs) via this domain. These results suggested that MYCL promotes reprogramming by regulating RNA processing.

\section{Results}

MYCL promotes reprogramming more efficiently than c-MYC. To compare the reprogramming phenotypes of MYCL and c-MYC, we used Sendai virus (SeV)-based reprogramming (CytoTune-iPS) and StemFit AK03N medium without bFGF (Fig. 1A). The SeV method has high reprogramming efficiency without genome integration, and c-MYC and MYCL SeV kits are already available ${ }^{15}$. The bFGF exclusion is based on the data in Supplementary Fig. S1. DMEM supplemented with 10\% FBS (DMEM + 10\%FBS) is the standard medium to induce reprogramming. We used DMEM $+10 \% \mathrm{FBS}$ when introducing the reprogramming factors, but after 7 days of reprogramming, we replated the cells and used StemFit AK03N without bFGF (03N (-)) from that point on. The MOI (multiplicity of infection) of each SeV was 20. To improve the reprogramming efficiency, we compared three media combinations (Supplementary Fig. S1A). The highest number of colonies was obtained using $03 \mathrm{~N}(-)$ during reprogramming and $03 \mathrm{~N}(+)$ after replating (Supplementary Fig. S1B). These results indicated that the $03 \mathrm{~N}(-)$ reprogramming condition in the first 7 days enhances the reprogramming efficiency compared to $03 \mathrm{~N}(+)$. We then examined the optimal MOI of $\mathrm{SeV}$ for the reprogramming (Supplementary Fig. S1C). A lower MOI induced more colonies (Supplementary Fig. S1D), indicating a higher reprogramming efficiency. Following these results, we applied $\mathrm{SeV}$ for the transduction at an MOI of 4.3 using 03N (-) during reprogramming.

Next, we conducted immunostaining to analyze the expression of TRA-1-60 from days 1 to 7 after the transduction (Fig. 1A). TRA-1-60 is a glycoprotein and major cell surface marker of hiPSCs and hESCs ${ }^{16}$. We quantified the results using a high-content imaging system, ArrayScan, because the cell number was small during $\mathrm{SeV}$ reprogramming for the first seven days, making flow cytometry challenging. On day 7 , we observed that c-MYC and MYCL induced a small cell mass to form colonies, but only the colonies induced by MYCL expressed TRA-1-60, while those induced by c-MYC looked like cell aggregations (Fig. 1B and Supplementary Fig. S2). Cell proliferation was highly increased in human dermal fibroblasts (HDFs) transduced with c-MYC compared to MYCL. On the other hand, the percentage of TRA-1-60 (+) cells increased more in MYCL-transduced HDFs on day 3 after the transduction (Fig. 1C). This difference may be because c-MYC has higher transformation activity than MYCL, which causes different phenotypes, especially cell proliferation ${ }^{10}$.

We confirmed these reprogramming phenotypes using episomal plasmid vector (EpiP) ${ }^{17}$ (Fig. 1D). SeV systems have a higher gene transfer efficiency, leading to more efficient reprogramming. However, we could modify the reprogramming vectors, which is useful for evaluating the molecular mechanism of c-MYC and MYCL, only when using the EpiP system.

Similar to the results with the SeV method, few colonies expressed TRA-1-60 in c-MYC-transfected HDFs (Fig. 1E and Supplementary Fig. S3A). However, the transfection of MYCL resulted in a higher percentage of TRA-1-60 (+) cells and lower cell proliferation than the transfection of c-MYC (Fig. 1F and Supplementary Fig. S3B). These differences between MYCL and c-MYC were more obvious with EpiP reprogramming than SeV reprogramming (Fig. 1C, F), probably because of differences in the gene transfer efficiency ${ }^{15,17}$, the expression of 
A

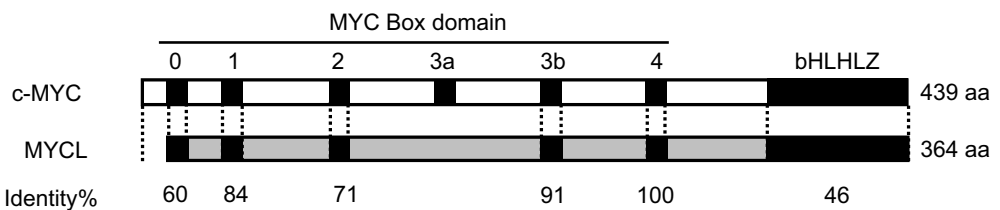

B

c-MYC
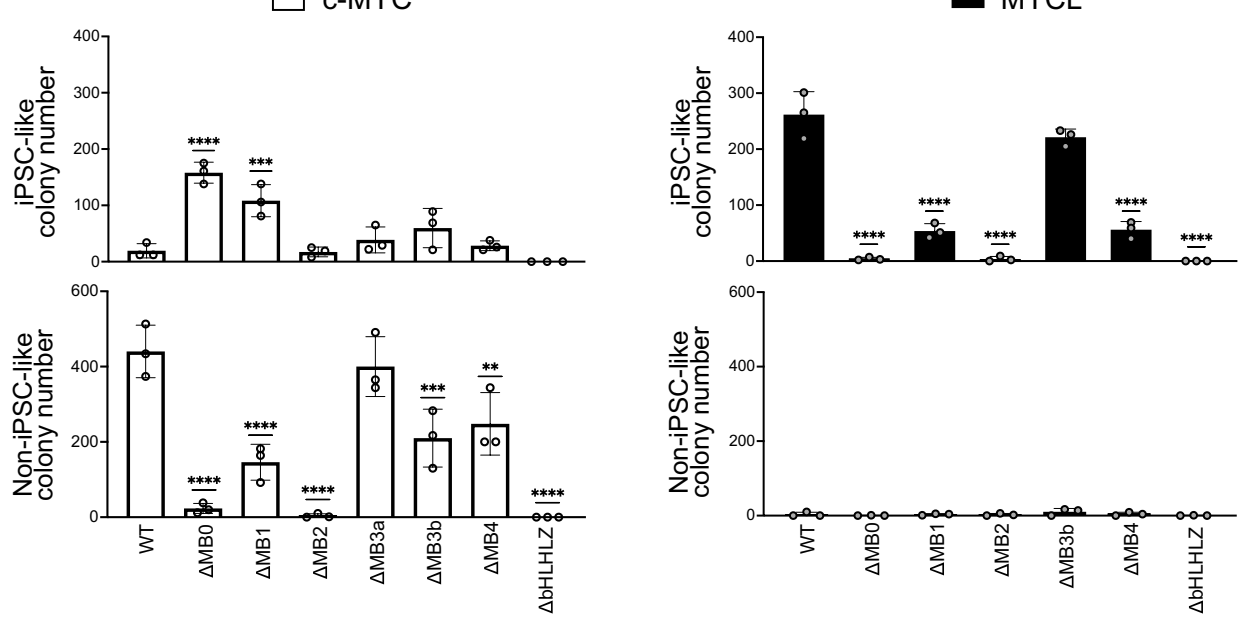

C
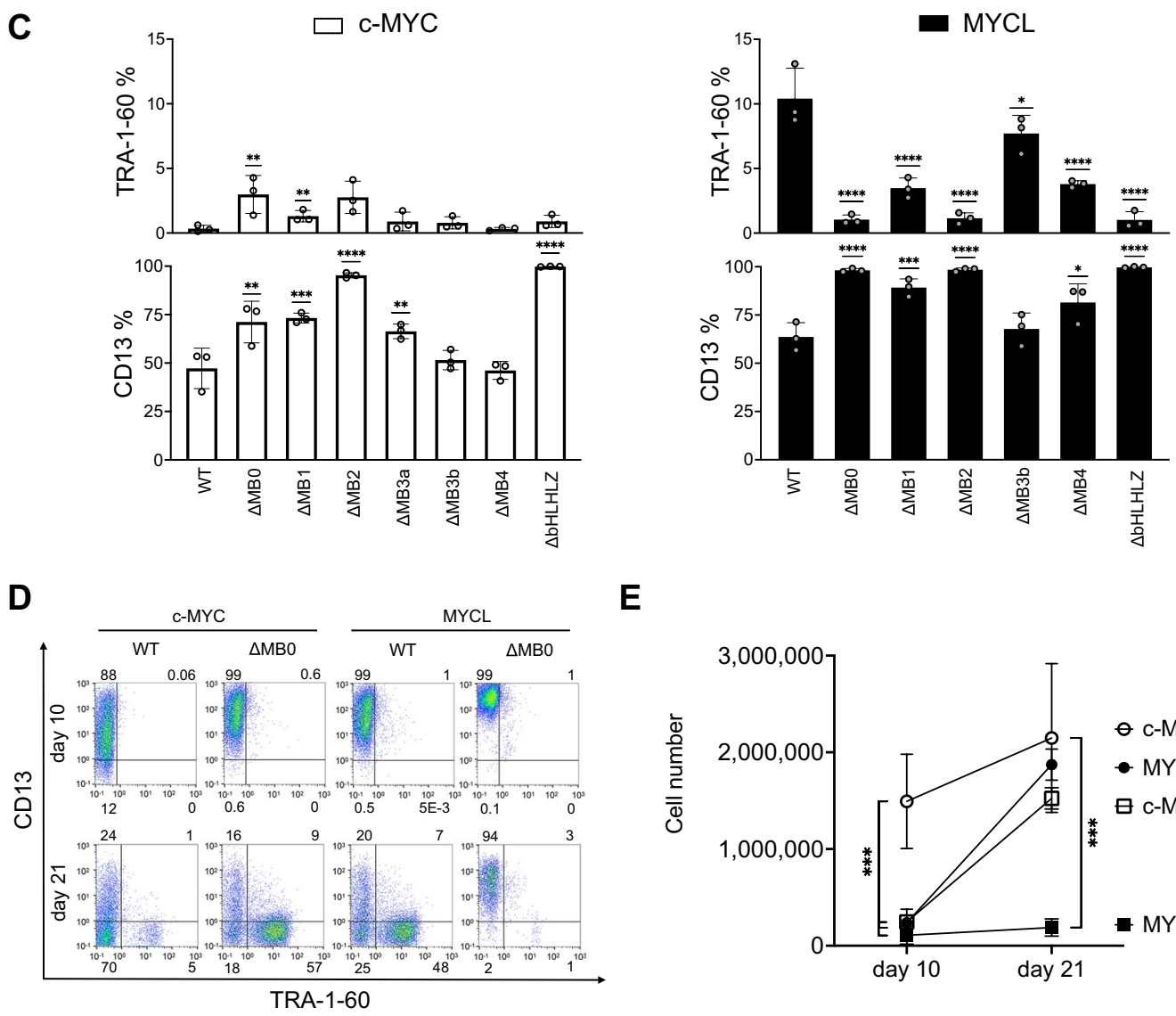

E

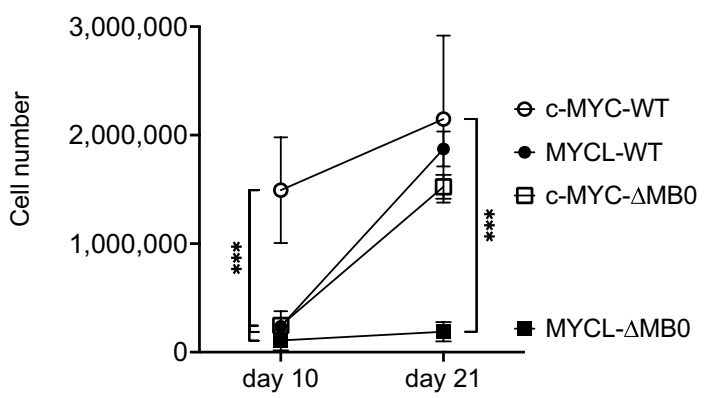


4Figure 2. MYC Box 0 and 2 domains are crucial for colony formation during reprogramming. (A) Schematic representation of WT c-MYC and MYCL protein. Black boxes show important domains for MYC function, including MB0, 1, 2, 3a (c-MYC only), 3b, 4, and basic-helix-loop-helix leucine zipper motif (bHLHLZ). The percentage of common amino acids in each MYC box domain between MYCL and c-MYC is shown (Identity\%). The numbers on the right indicate amino acid lengths. (B) Number of iPSC-like and non-iPSClike colonies transduced with EpiP including c-MYC-WT/mutants (left) or MYCL-WT/mutants (right) on day 21. Mean \pm SD values are shown. $n=3,{ }^{* *} p<0.01,{ }^{* * *} p<0.001$ and ${ }^{* * * *} p<0.0001$ by ordinary one-way ANOVA and Dunnett's test vs. WT. (C) Expression of TRA-1-60 (+) HDFs and CD13 (+) HDFs transduced with EpiP including c-MYC-WT/mutants (left) or MYCL-WT/mutants (right) on day 16. Mean \pm SD values are shown. $n=3,{ }^{*} p<0.05,{ }^{* *} p<0.01,{ }^{* *} p<0.001$ and ${ }^{* * * *} p<0.0001$ by ordinary one-way ANOVA and Dunnett's test vs. WT. (D) Representative flow cytometry images for TRA-1-60 and CD13 for HDFs transduced with EpiP including c-MYC-WT/ $\triangle \mathrm{MB} 0$ or MYCL-WT/ $\triangle \mathrm{MB} 010$ and 21 days after the transduction. Numbers indicate the expression percentage of each quadrant. (E) Proliferation of HDFs transduced with EpiP including c-MYC-WT/ $\triangle \mathrm{MB} 0$ or MYCL-WT/ $\mathrm{MB} 010$ and 21 days later. Mean \pm SD values are shown. $n=3,{ }^{* * *} p<0.001$ by ordinary one-way ANOVA and Dunnett's test vs. c-MYC-WT. The number of cells was counted using a Cell Counter model R1 (OLYMPUS).

the transfected factors, cell toxicity, and the time required for the iPSC-like colonies to appear: the SeV system requires about 7 days, but the EpiP system needs about 21 days based on our observations.

We found two types of colonies: "iPSC-like" and "non-iPSC-like" colonies. The iPSC-like colonies produced by MYCL were more flattened and showed a monolayered colony morphology, with each cell tightly packed and expressing TRA-1-60. The non-iPSC-like colonies produced by c-MYC showed a cell aggregation-like morphology, in which individual cells were irregularly aggregated and did not express TRA-1-60. We counted the number of iPSC-like and non-iPSC-like colonies on day 21 and found that c-MYC induced iPSC-like colonies as well as many non-iPSC-like colonies, but MYCL induced almost only iPSC-like colonies and more of them than c-MYC (Fig. 1G and Supplementary Fig. S3).

It has been reported that before the increase in the expression of TRA-1-60, a decrease in the expression of $\mathrm{CD} 13$, a marker of fibroblasts ${ }^{18}$, is observed in somatic cell reprogramming. Therefore, we confirmed the expression of CD13 during reprogramming. The percentage of CD13 (+) cells decreased daily in HDFs transduced with c-MYC or MYCL, but the number of CD13 (-) cells rapidly increased in c-MYC compared to MYCL (Fig. 1H and Supplementary Fig. S4). In particular, the CD13 (-) TRA-1-60 (-) population was larger on day 10 with c-MYC reprogramming than MYCL reprogramming, but the CD13 (-) TRA-1-60 (+) population from days 16 to 21 was larger with MYCL reprogramming (Supplementary Fig. S4). These results suggested that MYCL promotes TRA-1-60 (+) cells more than c-MYC, but c-MYC suppresses CD13 expression more than MYCL.

MYC Box 0 and 2 domains are crucial for colony formation during reprogramming. Next, we prepared domain deletion mutants to identify which domains in the N-terminus of MYC proteins influence reprogramming (Fig. 2A and Supplementary Fig. S5). We previously showed that a c-MYC mutant lacking transformation activity enhances the formation of iPSC-like colonies. This mutant has a point mutation in the transactivation domain of the N-terminal region, W135E (Fig. 2A and Supplementary Fig. S5B), but can bind to genomic $\mathrm{DNA}^{7}$. On the other hand, the bHLHLZ domain in the C-terminus region is a well-known binding domain of $\mathrm{MAX}^{19}$. Mutants in the C-terminus region prevent MYC proteins from binding to DNA and thus reprogramming ${ }^{7}$. Finally, we tested the reprogramming activities of these mutants using the EpiP reprogramming system because, as explained above, this method provided a clearer phenotype and was easier to manipulate than the $\mathrm{SeV}$ method.

The EpiP mutants were transfected into HDFs with other reprogramming factors, and the number of iPSClike and non-iPSC-like colonies was counted (Fig. 2B and Supplementary Fig. S6). c-MYC- $\Delta$ MB0 promoted the formation of iPSC-like colonies and inhibited the formation of non-iPSC-like colonies compared to c-MYC-WT. In contrast, MYCL- $\triangle \mathrm{MB} 0$ showed almost no ability to form iPSC-like colonies (Fig. 2B). We confirmed that the protein expression of each domain deletion mutant by western blotting showed no difference compared with c-MYC- or MYCL-WT (Supplementary Fig. S7 and S8). These results demonstrate that the MB0 domain has different functions in c-MYC and MYCL for reprogramming and that c-MYC- $\triangle \mathrm{MB} 0$ has a similar function as MYCL-WT.

Figure $2 \mathrm{~B}$ shows that c-MYC- $\triangle \mathrm{MB} 1$ promoted iPSC-like colony formation like c-MYC- $\Delta \mathrm{MB}$, but it also led to the formation of non-iPSC-like colonies. The formation of iPSC-like colonies by MYCL- $\triangle \mathrm{MB} 1$ was about a quarter that by MYCL-WT. Unlike c-MYC-WT, c-MYC- $\triangle \mathrm{MB} 2$ did not induce non-iPSC-like colonies, but it did induce a rate of iPSC-like colonies similar to c-MYC-WT. MYCL- $\triangle \mathrm{MB} 2$ showed little ability to form iPSC-like colonies, resembling MYCL- $\triangle \mathrm{MB}$. c-MYC- $\triangle \mathrm{MB} 3 \mathrm{a},-\triangle \mathrm{MB} 3 \mathrm{~b}$, and $-\Delta \mathrm{MB} 4$ had similar colony-forming activities as c-MYC-WT. MYCL- $\triangle$ MB3b showed the same reprogramming efficiency as MYCL-WT, but MYCL- $\Delta$ MB4 formed about the same small number of iPSC-like colonies as MYCL- $\triangle M B 1$. The $\triangle$ bHLHLZ mutants of both c-MYC and MYCL failed to induce colonies and were therefore considered to have lost MYC function completely. Thus, the results indicate that in $\mathrm{c}-\mathrm{MYC}$, the MB0 and MB2 domains are repressive for iPSC-like colony formation, but in MYCL, they are promotive. Other domains also influenced the colony formation efficiency, but the effect was small.

Next, we analyzed the effect of the MYC-deletion mutants on the expression of TRA-1-60 and CD13 by flow cytometry 16 days after the start of reprogramming (Fig. 2C). Mutants that increased the number of iPSC-like colonies also increased the expression of TRA-1-60, while those that reduced the number of iPSC-like colonies lowered the TRA-1-60 expression (Fig. 2C and Supplementary Fig. S9). c-MYC-WT showed little TRA-1-60 
expression, whereas c-MYC- $\triangle \mathrm{MB} 0$ upregulated the expression. MYCL- $\Delta \mathrm{MB} 0$, unlike MYCL-WT, failed to upregulate the expression of TRA-1-60. The CD13 expression was also correlated with colony formation. In c-MYC, a significant decrease in CD13 expression was observed for mutants that promoted non-iPSC-like colony formation. As for MYCL, only a slight decrease in CD13 expression was observed for mutants that promoted iPSC-like colony formation. From these results, we concluded that the MB0 domain is essential for the function of MYC in reprogramming but functions differently between c-MYC and MYCL.

To analyze the function of the MB0 domain in more detail, we analyzed the expression of TRA-1-60 and CD13 10 and 21 days after the start of reprogramming by flow cytometry (Fig. 2D). In the case of c-MYC-WT, there was a strong decrease in CD13 expression on day 10, and most cells were CD13 negative on day 21. In the cases of $\mathrm{c}-\mathrm{MYC}-\triangle \mathrm{MB} 0$ and MYCL-WT, there was a slight decrease in CD13 expression on day 10, and more than half of cells were expressing TRA-1-60 on day 21. Finally, in the case of MYCL- $\triangle M B 0$, there was no change in CD13 or TRA-1-60 expression. More study is needed to determine how CD13 is regulated by c-MYC and MYCL.

Additionally, c-MYC-WT showed higher cell proliferation on day 10, but c-MYC- $\triangle$ MB0 resulted in a lower cell proliferation comparable more with MYCL-WT than with c-MYC-WT on day 10 (Fig. 2E). We attributed this effect to the lost transformation activity of c-MYC- $\triangle \mathrm{MB} 0$. From days 10 to 21 , the cell proliferation increased significantly in c-MYC- $\triangle \mathrm{MB} 0$ and MYCL-WT, and a concomitant increase in the CD13 (-) TRA-1-60 (+) population was observed (Fig. 2D, E). These observations suggest that the number of cells that were reprogrammed increased rapidly with c-MYC- $\mathrm{MB} 0$ and MYCL-WT. With c-MYC-WT, the cell proliferation continued until day 21. However, the CD13 (-) TRA-1-60 (+) population hardly increased (Fig. 2D), indicating that these cells were not reprogramming but changing to other highly proliferative cell types. From these results, we concluded that the MB0 domain functions negatively in c-MYC and positively in MYCL for reprogramming.

MYCL regulates cytoskeleton- and cell adhesion-related proteins during reprogramming via the MBO domain. To confirm which genes are regulated by the MYCL MB0 domain in reprogramming, we analyzed protein expressions during reprogramming because it was reported that gene expressions do not correlate well with protein expressions ${ }^{20}$. We performed a comprehensive analysis of expressed proteins during reprogramming induced by $\mathrm{c}-\mathrm{MYC}$ and MYCL WT and $\triangle \mathrm{MB} 0$ mutants. We used SeV-reprogramming HDFs on days 3, 5, and 7 days and EpiP-reprogramming HDFs on day 10 as samples for mass spectrometry (MS) (Fig. 3A) because the percentage of TRA-1-60 (+) cells was much higher with SeV than with EpiP for observations up to day 7 (Fig. 1C, F). There was more than a two-fold increase in the expression of $520(\mathrm{SeV})$ and 128 (EpiP) proteins with MYCL-WT reprogramming compared to c-MYC-WT reprogramming (Fig. 3B, groups (i) and (ii), respectively) and 183 (EpiP) proteins with c-MYC- $\triangle \mathrm{MB} 0$ reprogramming compared to c-MYC-WT reprogramming (Fig. 3B, group (iii)). Overall, we identified 18 proteins common to the three groups (Fig. 3B, group (iv)). Then, we applied a Gene Ontology (GO) analysis using DAVID and detected enriched terms during reprogramming ${ }^{21,22}$ (Fig. 3C, D, and Table 1), finding cytoskeleton- and cell adhesion-related proteins are involved in the promotion of reprogramming by MYCL-WT. The same analysis was performed to identify proteins whose expression was upregulated by c-MYC-WT compared with MYCL-WT and c-MYC- $\triangle$ MB0 (Supplementary Fig. S10A and Table 2). These proteins were associated with the proliferation of non-iPSC-like colonies. We found that c-MYC-WT regulates proteins involved in cell proliferation, such as the cell cycle and DNA replication. To understand the function of the MB0 domain in reprogramming, MS analysis was applied to HDF samples transfected with MYCL-WT, MYCL- $\triangle \mathrm{MB} 0$, or c-MYC- $\triangle \mathrm{MB} 0$ (Supplementary Fig. S10B and Table 3). $\mathrm{GO}$ analysis indicated that these proteins were associated with cell adhesion and RNA processing.

We also compared phosphorylated proteins during SeV reprogramming with MYCL and c-MYC. In total, there was more than a two-fold relative increase of 17 phosphorylated proteins with MYCL-WT and 132 phosphorylated proteins with c-MYC-WT. The GO analysis indicated that the phosphorylated proteins increased by MYCL included cytoskeleton-related proteins and those increased by c-MYC included transcription-related proteins (Supplementary Fig. S11).

MYCL regulates RNA processing-related proteins during reprogramming via the MB2 domain. Our analysis also revealed that, along with the MYCL MB0 domain, the MYCL MB2 domain is important for reprogramming (Fig. 2B). It has been reported that the c-MYC MB2 domain is involved in transformation activity, and tryptophan 135 within the MB2 domain is necessary for this activity ${ }^{10}$. MYCL also has a tryptophan residue within its MB2 domain but little transformation activity ${ }^{23}$. We hypothesized that this domain in MYCL has reprogramming function. We therefore produced a mutant in which tryptophan 96 was substituted with glutamate (W96E). This tryptophan is equivalent to tryptophan 135 in c-MYC (Fig. 4A and Supplementary Fig. S5B). We confirmed the expression of MYCL-W96E by western blotting (Supplementary Fig. S12). Next, we examined the effect of MYCL-W96E for reprogramming. HDFs were transfected with reprogramming factors including MYCL-WT or -W96E. MYCL-W96E could not induce iPSC-like colonies, suggesting tryptophan 96 is crucial for reprogramming (Fig. 4B, C). We thus hypothesized that the residue might be important for MYCL to bind to other proteins. To identify the binding proteins, we produced GST-fusion recombinant proteins of the MYCL MB2 domain (Fig. 4A). GST-MYCL-MB2-WT or -W96E proteins were immobilized on glutathione Sepharose, and affinity columns were prepared. Cell lysates were applied to the column, and, after washing, the bound proteins were eluted. We used the cell lysates from reprogramming HDFs, but since it was difficult to collect a large amount, we also used cell lysates from hiPSCs. The reason for using the hiPSC lysates is that many of the proteins expressed in reprogramming HDFs are highly expressed in hiPSCs as well ${ }^{16,24-27}$.

We identified 31 candidate proteins that bind to the MB2 domain of MYCL-WT but not of MYCL-W96E during reprogramming in the HDF lysates (Fig. 4D and Table 4). Of those 31 proteins, 25 proteins were also identified using hiPSC lysates, and 23 were RNA-binding proteins (RBPs; Fig. 4D, genes written in blue). Six 
A

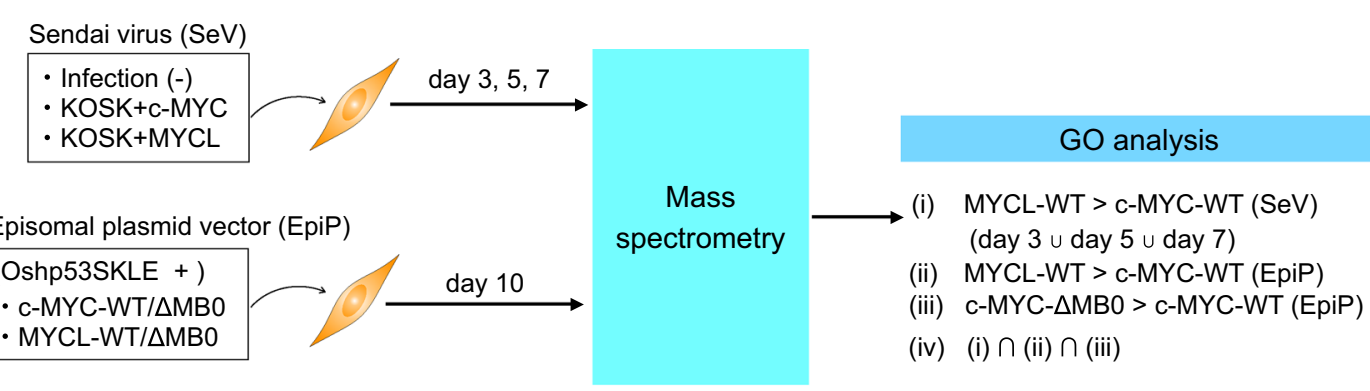

B

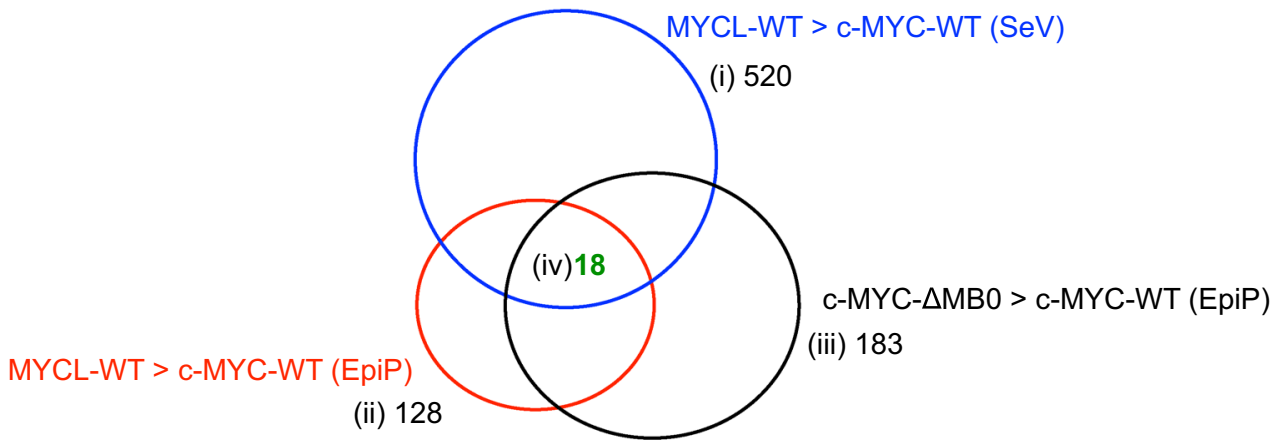

C

$\mathrm{GO}$ analysis

Group

(i)
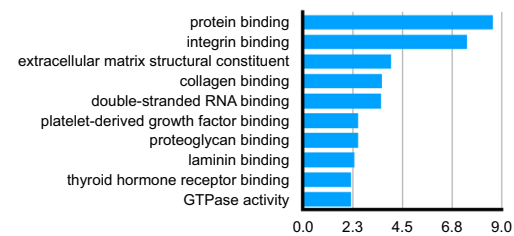

(ii)

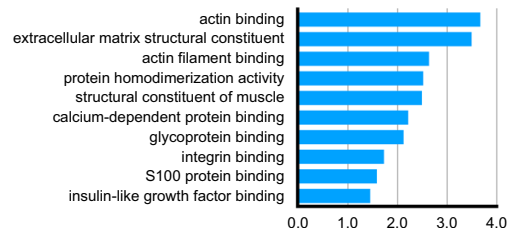

(iii)

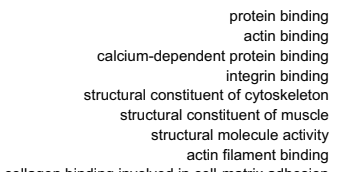

(iv)

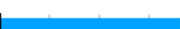

$$
-
$$$$
\text { D }
$$

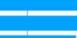

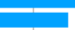

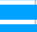

protease binding

structural constituent of muscle

actin binding

structural constituent of cytoskeleton

actin filament binding

structural molecule activity

calcium-dependent protein binding

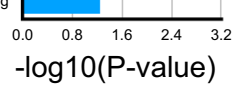

D Group

(i)

GO analysis KEGG pathway

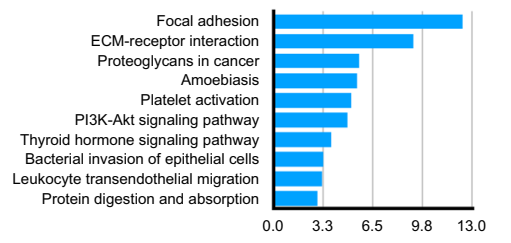

(ii)

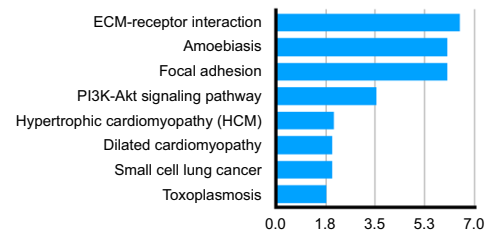

(iii)

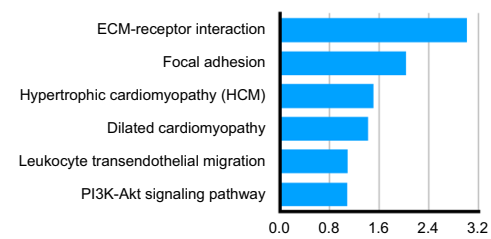

(iv)

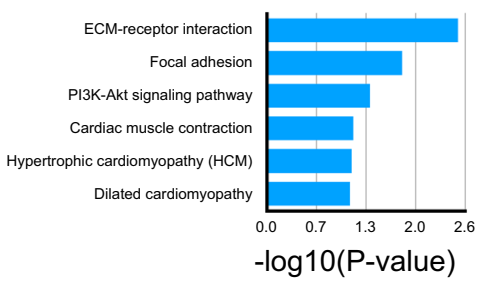

Figure 3. MYCL regulates cytoskeleton- and cell adhesion-related proteins during reprogramming via the MB0 domain. (A) Schematic of the mass spectrometry (MS) and GO analysis (DAVID). (B) Venn diagram of upregulated proteins during iPSC-like colony formation. (C) Molecular functions from the GO analysis of the four groups in (B). (D) KEGG pathways from the GO analysis of the four groups in (B). 
(i) Proteins enriched more than two-fold in MYCL-WT compared with c-MYC-WT (SeV)

\begin{tabular}{|c|c|c|c|c|c|}
\hline NRP1 & PMEL & IQCH & ZNF507 & C1QTNF3 & GLIPR2 \\
\hline ING1 & THOC7 & RPAIN & DGCR8 & KRAS & PIPOX \\
\hline CCNL2 & ACOT8 & KCNMA1 & TTC38 & MRI1 & DLGAP5 \\
\hline CHPF2 & P4HA1 & HMCN1 & STRA13 & SMG5 & FAM83D \\
\hline MTFR1L & TSPYL1 & CROT & PLPP1 & PRKACB & FSD1 \\
\hline REPIN1 & MKLN1 & CPS1 & MDP1 & ZWILCH & ST6GAL1 \\
\hline MCL1 & SRR & FAM134A & USP34 & CEP41 & MATN2 \\
\hline AQP1 & PPL & SON & ARL14EP & ACSS3 & KLHL11 \\
\hline FAIM & МСMBP & COL1A1 & MOCS3 & SHC1 & DPT \\
\hline VCPIP1 & TPM2 & CLDN7 & C18orf32 & SAG & POSTN \\
\hline AKAP11 & AMDHD2 & AHCYL2 & MASTL & MAP3K2 & COPZ2 \\
\hline ARFGEF3 & HBA1,HBA2 & S100P & CCNYL1 & RALGAPB & ACTR1B \\
\hline PIAS4 & PFKFB3 & FAM134C & SDSL & PPIC & NR3C1 \\
\hline FYN & SPAST & MAP4K2 & COQ3 & CENPV & HERC2 \\
\hline CDS2 & TADA2B & XPC & MX1 & PCSK9 & SDPR \\
\hline CEP131 & FEM1A & ACTG1 & $\mathrm{TNC}$ & ITPR3 & GNPTG \\
\hline SH3BGRL2 & QSOX1 & LSM4 & FBXL18 & SH3BP5L & FARP2 \\
\hline ZIC5 & PASK & FLYWCH2 & TMEM119 & FAP & AGTPBP1 \\
\hline ANKIB1 & EDEM3 & PANX1 & CCDC28A & DDX58 & FOXK2 \\
\hline ERICH1 & KIAA1211 & ZMYM4 & FN1 & ARSA & CSNK1E \\
\hline MTRR & NCOA3 & PATZ1 & UBE2S & DDB2 & CCDC68 \\
\hline POLG2 & C10orf76 & ADIRF & CALD1 & RALGAPA1 & NUDT9 \\
\hline YAE1D1 & C14orf142 & TSPAN14 & PTGIS & FAM208A & PANK1 \\
\hline TCN2 & TAGLN & ALG8 & THAP11 & NFIC & TMEM165 \\
\hline BLOC1S6 & FAM21A & NCOR2 & COL12A1 & TGFBI & CRELD1 \\
\hline MARH5 & CNOT8 & RANGRF & MED16 & CDA & GULP1 \\
\hline WDR54 & MET & NOA1 & PRKG1 & CHMP1A & SHARPIN \\
\hline RRP8 & \begin{tabular}{|l|} 
TBC1D7 \\
\end{tabular} & CPQ & IFIT1 & THBS1 & HSDL2 \\
\hline GORAB & TRAF6 & AHDC1 & DDX60 & NDUFB6 & ARHGEF6 \\
\hline CERCAM & NPEPL1 & GPR107 & MAP3K15 & MRS2 & ELP3 \\
\hline TPM1 & COMMD8 & MED4 & HACL1 & IGFBP3 & HTRA1 \\
\hline CD99L2 & PEX16 & GINS4 & DSCR3 & UBE2G1 & EIF4EBP1 \\
\hline DYNC1I2 & ACTN1 & YPEL5 & SMG6 & ITGB4 & PTGES \\
\hline TPK1 & REEP6 & PMF1 & PTBP2 & IFIT2 & PUM1 \\
\hline DYNC2H1 & KDELR3 & VIPAS39 & KIF1B & EMILIN2 & CRIP2 \\
\hline HIGD2A & C7orf26 & DNM2 & MMP2 & KANK2 & DHX30 \\
\hline RAP1B & DNAJB5 & MRPL33 & SPANXA2-OT1 & PIR & SDCBP \\
\hline HMGXB4 & POLG & FOXK1 & PEX1 & LGALS8 & LAMC1 \\
\hline DNAH6 & PDIA4 & MTMR14 & S100A14 & CNTLN & SLC25A32 \\
\hline TMED4 & SPARC & GBP1 & CNN2 & GCC1 & CTHRC1 \\
\hline STAU2 & SUPV3L1 & DNAJC16 & KIAA0430 & CASP4 & NID2 \\
\hline FAM69C & TIMP2 & OGFOD3 & EED & DCX & PRNP \\
\hline KCTD15 & GSPT2 & PCNT & STARD4 & OTUD7B & \begin{tabular}{|l|} 
PРP3CC \\
\end{tabular} \\
\hline WDR35 & CTSZ & SLC15A4 & BASP1 & SLC44A1 & AKR1C2 \\
\hline $\mathrm{COA} 3$ & RAB2B & GNA12 & OPA3 & INPP5A & GAP43 \\
\hline CAAP1 & VWA8 & PALM & KRT17 & MIEF2 & IKBKB \\
\hline C1orf198 & BUB1 & ZBTB7A & CD248 & ACOX1 & \begin{tabular}{|l|} 
DNAJA4 \\
\end{tabular} \\
\hline CNN1 & ANAPC4 & LOX & LAMA5 & COL2A1 & KRT6A \\
\hline LRRC41 & COL6A3 & CABIN1 & ECM1 & MED8 & KIF21A \\
\hline NOL8 & SLC30A5 & COL16A1 & TWISTNB & GREM1 & ICAM1 \\
\hline OSBPL11 & TBC1D15 & HORMAD2 & EPHA2 & MRPL51 & B3GALT6 \\
\hline USP9Y & VKORC1 & ETNK1 & MACF1 & STAG3 & SH3KBP1 \\
\hline BCAR1 & KHDRBS3 & TLE3 & IGF2 & STARD3NL & CTDSPL2 \\
\hline FIBP & RANBP10 & IFT74 & SERPINB2 & SAMD9 & FZD7 \\
\hline LGALS1 & CSRP1 & FBLN1 & SERPINF1 & SHCBP1 & TUBG1 \\
\hline CAPN5 & PTK7 & PLAUR & ZNF185 & SGF29 & RASA3 \\
\hline ACSF3 & DNA2 & PRSS23 & PKP3 & GDAP1 & CAV2 \\
\hline
\end{tabular}


(i) Proteins enriched more than two-fold in MYCL-WT compared with c-MYC-WT (SeV)

\begin{tabular}{|c|c|c|c|c|c|}
\hline FBXO2 & CCND1 & SLC34A3 & KYNU & RBM23 & ACBD7 \\
\hline MAP2 & MIC13 & IFI44 & PIK3CA & KLC4 & ODR4 \\
\hline GATC & TANGO6 & MITD1 & ATL1 & ANAPC13 & SP100 \\
\hline MYL9 & COL6A2 & PPIL2 & MPDZ & CCBL1 & TGM2 \\
\hline TGS1 & CDYL & KRT10 & SNX32 & OGN & FMNL3 \\
\hline LAMB1 & CSRP2 & MON2 & FAM127A & WDR4 & KRT14 \\
\hline BEND3 & TRIM21 & RPS6KA4 & LENG8 & SPRR3 & FRG1 \\
\hline KLK14 & CDC34 & ASAP2 & TAP2 & NEXN & INPP5B \\
\hline TPM4 & PPFIA1 & KRT16 & LIMK1 & HOOK2 & PPP2R2D \\
\hline CPLX1 & SUN1 & WDR73 & WWC3 & SMG7 & COL1A2 \\
\hline CCDC92 & MYCL & DESI2 & FYCO1 & RAC2 & SEMA7A \\
\hline TIMP3 & PKD1L3 & COL5A1 & PYURF & DPYSL4 & SNX24 \\
\hline SERPINB8 & FBXO3 & RNF31 & AKTIP & BAZ2A & UGGT2 \\
\hline HSPB1 & CBX2 & IFIT3 & QPCTL & Clorf50 & CAV1 \\
\hline CD58 & COL11A2 & ISG15 & RPL26L1 & TYW3 & CD44 \\
\hline PARP2 & GOLT1B & FOSL1 & GRB7 & CREG1 & HOMER3 \\
\hline HSPB6 & ABR & NID1 & ECT2 & RAP2A & NDRG1 \\
\hline COL5A2 & AURKA & GSDMD & ENG & WDR55 & WNT5A \\
\hline MRGBP & EP300 & MAU2 & CHST14 & GHDC & NPHP3 \\
\hline FABP3 & ANPEP & ARHGDIB & BST1 & NABP2 & SIRT5 \\
\hline DTX3L & HAUS7 & LTBP2 & CLINT1 & PXMP4 & DPY19L1 \\
\hline ARL5A & RNF113A & CRBN & GGA1 & UBE2F & GPNMB \\
\hline NT5E & CILP & MROH2B & SEPT5 & ILF3 & TANC1 \\
\hline STX3 & NOTCH3 & PLCG2 & ARFIP1 & NCOA5 & QSOX2 \\
\hline SLC2A1 & S100A6 & CDCA5 & CALHM2 & KDM4B & TIMELESS \\
\hline F13A1 & COMMD9 & REN & MECP2 & TNXB & ZYG11B \\
\hline AHNAK2 & RDH10 & CLIC3 & MME & SLC39A14 & GGCX \\
\hline S100A4 & ZCCHC6 & CD9 & CD82 & LTBP1 & STK11 \\
\hline UAP1L1 & MED12 & PXN & GOSR2 & B3GNT5 & ABI3BP \\
\hline ITGA2 & OASL & CTSK & VAMP8 & & \\
\hline
\end{tabular}

(ii) Proteins enriched more than two-fold in MYCL-WT compared with c-MYC-WT (EpiP)

\begin{tabular}{|l|l|l|l|l|l|}
\hline IGFBP3 & CLU & CTHRC1 & CDH13 & CAVIN3 & CCDC80 \\
\hline GLIPR2 & GBP1 & FLNA & LAMB1 & S100A6 & COL5A2 \\
\hline CNN2 & ITGA11 & SH3BGRL3 & CPQ & PRSS23 & LAMC1 \\
\hline NEGR1 & LAMA5 & IFIT2 & LGALS1 & HTRA1 & TAGLN \\
\hline TPM2 & HSPB1 & EHD2 & VIM & CAVIN1 & CSRP1 \\
\hline TPM1 & TGFBI & S100A11 & S100A4 & COL5A1 & NEXN \\
\hline MOXD1 & SLFN5 & NME2P1 & FNBP1 & PLSCR3 & ATPAF2 \\
\hline ZFYVE16 & HSBP1 & DGKA & PLCD1 & ABI3BP & ANXA3 \\
\hline TCF3 & PLCB4 & SLC34A3 & TMEM192 & ADAMTSL1 & ENDOD1 \\
\hline PTGES & OSBPL9 & LRRFIP1 & GDPD2 & CRYAB & SETDB1 \\
\hline ST6GALNAC1 & SMPD1 & SETMAR & SORBS3 & B4GALT4 & CHKB \\
\hline RNF14 & TNXB & AHNAK2 & DCTN3 & PTBP2 & EHBP1L1 \\
\hline TERF2IP & ARMC8 & PRC1 & UQCC3 & PTP4A2 & TTYH3 \\
\hline MYCL & BCAT2 & ELN & CRMP1 & MX2 & SGCE \\
\hline HABP2 & TXNIP & RMND5A & TIMP1 & PITPNA & DDX49 \\
\hline CHIC1 & COL6A3 & IMPACT & RAB11B & TMSB4X & SNX7 \\
\hline DDR2 & KRT5 & PLEKHO2 & SMTN & LIPG & RGS3 \\
\hline GREM1 & COL5A1 & HNRNPDL & LMOD1 & MAP4K4 & PPP3CB \\
\hline QTRT2 & ARID1A & KRT17 & SELENOM & CYP1B1 & CD151 \\
\hline MT1X & CTNNA2 & KRT6A & MBD5 & RAP1B & R3HCC1 \\
\hline VPS37A & STAM2 & CTSL & ITGA1 & PAIP1 & DHRS4 \\
\hline MTPAP & CDK2 & & & & \\
\hline & & & Bold value: $p<0$ 05 & & \\
\hline (iii) Proteins enriched more than two-fold in c-MYC- 4 MB0 compared with c-MYC-WT (EpiP) \\
\hline ITGA1 & PSMF1 & FNBP1 & BCAT2 & DGKA & YIPF3 \\
\hline Continued & & & & \\
\hline
\end{tabular}


(iii) Proteins enriched more than two-fold in c-MYC- $\triangle \mathrm{MBO}$ compared with c-MYC-WT (EpiP)

\begin{tabular}{|c|c|c|c|c|c|}
\hline MBD5 & KRT5 & S100A6 & MOXD1 & RMND5A & PPIL3 \\
\hline TAP1 & PAIP2 & OXR1 & MMP2 & SCLY & TBC1D10B \\
\hline CYP1B1 & PPDPF & TPM1 & PLSCR3 & SEMA7A & РРРЗСВ \\
\hline RNF115 & TMEM192 & IGFBP3 & MBD2 & RBM6 & ITPRIP \\
\hline SPIRE2 & OSBPL9 & TPM2 & \begin{tabular}{|l|} 
TERF2IP \\
\end{tabular} & DIP2B & RAB11B \\
\hline EDC3 & SMPD1 & DHRS4 & LMOD1 & OS9 & CPQ \\
\hline COG3 & KRT6A & HSBP1 & \begin{tabular}{|l|} 
LRRFIP1 \\
\end{tabular} & CHCHD6 & FNTB \\
\hline CD151 & EXOC6B & MROH1 & COL6A3 & AHNAK2 & BCL2L13 \\
\hline RPS6KC1 & NCOR2 & UQCC3 & SELENOM & COG4 & DNAJC17 \\
\hline PRC1 & CDK2 & INTS1 & ATPAF2 & ENDOD1 & STK10 \\
\hline DUSP23 & PTGES & CCDC50 & TIMP1 & TRIM56 & YAP1 \\
\hline CHIC1 & EHBP1L1 & OLFML3 & CLU & RAC2 & EHD2 \\
\hline SDC1 & DNAJC19 & CTNNA2 & CDH13 & ITPA & PITPNA \\
\hline SNAPIN & PTPN14 & LEMD3 & SEC24A & CCDC9 & MROH9 \\
\hline PAIP1 & ANXA3 & BAG3 & MAP3K20 & ELN & NEXN \\
\hline STAM2 & IMPACT & ZFYVE16 & TNXB & RMDN2 & CRMP1 \\
\hline HABP2 & TIMP2 & MYO1D & TRAPPC2L & LAMA5 & CWC22 \\
\hline DLGAP4 & TANC1 & DCTN3 & \begin{tabular}{|l|} 
CAVIN3 \\
\end{tabular} & \begin{tabular}{|l|} 
PPIP5K2 \\
\end{tabular} & LMAN2L \\
\hline ELP4 & SRSF4 & KRT17 & IFIT2 & ERI1 & EEF1AKNMT \\
\hline SMTN & NEGR1 & HNRNPDL & ARID1A & PARP12 & SCRN3 \\
\hline WDR4 & UBAP1 & SLFN5 & NCOA5 & PHRF1 & SPARC \\
\hline CAV1 & KRT16 & ERCC2 & MYO1C & L2HGDH & \begin{tabular}{|l|} 
LPCAT3 \\
\end{tabular} \\
\hline ITGA11 & NELFA & PXDC1 & IL12RB2 & EIPR1 & DPP7 \\
\hline MCAM & COG1 & POLR3C & HSPB1 & CAVIN1 & GLIPR2 \\
\hline PIKFYVE & \begin{tabular}{|l|} 
C1orf198 \\
\end{tabular} & SCPEP1 & PALM & NKRF & CNN1 \\
\hline RHOB & CUL4B & CCDC80 & ARL2BP & GNG12 & BMP1 \\
\hline STIM1 & \begin{tabular}{|l|} 
VKORC1 \\
\end{tabular} & CERCAM & CFAP74 & ERGIC3 & S100A4 \\
\hline TAGLN & MRPS6 & KIF4A & P3H4 & ALKBH4 & PTP4A2 \\
\hline ELMO2 & CRYAB & SLC27A1 & MAEA & GRB10 & THY1 \\
\hline AP1S2 & ZNF579 & SLC33A1 & & & \\
\hline \multicolumn{6}{|c|}{ (iv) Commonly enriched proteins in (i) and (ii) and (iii) } \\
\hline \multicolumn{6}{|l|}{ KRT6A } \\
\hline \multicolumn{6}{|l|}{ PTGES } \\
\hline \multicolumn{6}{|l|}{ S100A6 } \\
\hline \multicolumn{6}{|l|}{ TPM1 } \\
\hline \multicolumn{6}{|l|}{ IGFBP3 } \\
\hline \multicolumn{6}{|l|}{ TPM2 } \\
\hline \multicolumn{6}{|l|}{ KRT17 } \\
\hline \multicolumn{6}{|l|}{ COL6A3 } \\
\hline \multicolumn{6}{|l|}{ TNXB } \\
\hline \multicolumn{6}{|l|}{ IFIT2 } \\
\hline \multicolumn{6}{|l|}{ AHNAK2 } \\
\hline \multicolumn{6}{|l|}{ LAMA5 } \\
\hline \multicolumn{6}{|l|}{ CPQ } \\
\hline \multicolumn{6}{|l|}{ NEXN } \\
\hline \multicolumn{6}{|l|}{ TAGLN } \\
\hline \multicolumn{6}{|l|}{ HSPB1 } \\
\hline \multicolumn{6}{|l|}{ GLIPR2 } \\
\hline S100A4 & & & & & \\
\hline
\end{tabular}

Table 1. MS analysis of identified proteins in cells reprogrammed by MYCL- or c-MYC- $\triangle \mathrm{MB} 0$. Four groups are described: (i) proteins whose peptide counts increased more than two-fold in MYCL-WT/HDFs compared with c-MYC-WT/HDFs using SeV on day 3, 5, or 7; (ii) proteins whose peptide counts increased more than two-fold in MYCL-WT compared with c-MYC-WT using EpiP; (iii) proteins whose peptide counts increased more than two-fold in c-MYC- $\triangle \mathrm{MB} 0$ compared with c-MYC-WT using EpiP; and (iv) commonly identified proteins. Bold fonts in the group (ii) indicate identified proteins with $p<0.05$ (two-sample paired $t$-test). $n=3$ for EpiP reprogramming. 
(i) Proteins enriched more than two-fold in c-MYC-WT compared with MYCL-WT (SeV)

\begin{tabular}{|c|c|c|c|c|c|}
\hline ATXN7L3B & TIMM21 & SLC2A3 & CA14 & CRLF3 & SYT6 \\
\hline TMEM161A & MTM1 & METTL15 & NKAP & CDS2 & MRS2 \\
\hline MARS2 & ERCC2 & TDP1 & MFAP4 & ANAPC16 & CARS2 \\
\hline NOLC1 & IGHMBP2 & MRPL34 & FECH & PARP2 & ING1 \\
\hline ADNP2 & STEAP3 & AK6 & PDZD8 & EPB41L5 & PEX16 \\
\hline ZER1 & CKS1B & GGPS1 & DBNDD1 & MIEF1 & FUCA1 \\
\hline ADSSL1 & POTEJ & TMEM209 & CCNL2 & TOP3A & ULK1 \\
\hline MGA & FAM162A & AMMECR1 & ISG20L2 & CEP78 & NOM1 \\
\hline PAPD4 & PROCR & IFRD2 & LRRC41 & UBR3 & PHF3 \\
\hline RIN1 & SPPL2B & ARAF & DNM2 & HPS5 & PSEN1 \\
\hline PARD3 & ARHGEF16 & RHPN2 & PRPF18 & SEMA4C & RPUSD3 \\
\hline NYNRIN & ARHGEF7 & VRTN & PHF10 & DMD & \begin{tabular}{|l|} 
RPL26L1 \\
\end{tabular} \\
\hline RANBP6 & CNOT4 & TSPYL5 & CDC25C & REEP4 & FADD \\
\hline INPP5F & ZBTB7A & GPN3 & RBPMS2 & BRAF & ORC6 \\
\hline CACNA2D2 & AP1B1 & NCAPD3 & RRP8 & MASTL & POLR2M \\
\hline CASC3 & NCL & C1orf174 & LRRC14 & SLC27A3 & ACSF3 \\
\hline DHRS11 & RBM23 & WDR55 & CAMK4 & NDRG3 & ALS2 \\
\hline NOVA1 & SOX3 & CLCN7 & EHMT1 & C7orf26 & NSUN5 \\
\hline NMRAL1 & STK25 & NFKB2 & OSBPL1A & VPS37B & RAD23A \\
\hline HS2ST1 & LYAR & PHKA1 & SDC4 & MGST2 & SNTB1 \\
\hline MEN1 & WDR4 & DDX28 & C1orf198 & AKAP9 & COQ9 \\
\hline STYX & PHF5A & PCDH1 & \begin{tabular}{|l|} 
TMSB4X \\
\end{tabular} & AP1G2 & MYO1G \\
\hline UCKL1 & APC & TBC1D15 & FASTKD1 & APCDD1L & MARH5 \\
\hline ULK3 & LONP2 & SETD1A & ETFDH & ANKS1A & LRP8 \\
\hline PALD1 & ANAPC5 & CARMIL1 & GATM & PANX1 & NME3 \\
\hline UBA52 & ZNF806 & NCOR2 & DVL2 & CTDP1 & PHKB \\
\hline GINS3 & DNPH1 & CDCA5 & BCKDK & TTF1 & TGFBRAP1 \\
\hline HAUS2 & HMGCR & SNCA & KLHDC4 & ТВР & AP1M2 \\
\hline $\mathrm{E} 2 \mathrm{~F} 4$ & AFAP1L1 & ZMYM6NB & N4BP2 & TRIT1 & CCDC134 \\
\hline ATL1 & INTS6 & CHD8 & SPINT2 & RASA2 & NCK2 \\
\hline MAL2 & ATAD3A & SLC25A32 & LSAMP & ACOT8 & KIFAP3 \\
\hline JARID2 & CLSTN1 & USP36 & PTGIS & PIAS4 & TMEM41B \\
\hline SEC14L1 & TUBGCP4 & GEMIN8 & VWA9 & RPP25L & NRBP2 \\
\hline DOLPP1 & WARS2 & PLEKHA6 & MRGBP & ZCCHC6 & ZFP36L1 \\
\hline SLC4A7 & SCARB1 & ARID1B & PMF1 & XXYLT1 & ANKRD50 \\
\hline MT-CO1 & MET & RBM47 & LIN28B & EXD2 & GORAB \\
\hline GCSH & PLTP & PRKAB1 & CUTC & SDSL & FARS2 \\
\hline LRRC8E & ARHGAP12 & FBXW9 & PMS2 & NAA30 & STRA13 \\
\hline FASTKD5 & ZCCHC10 & TTK & BNC2 & COX16 & BCS1L \\
\hline NDE1 & STX3 & LARP1B & PTCD1 & TPD52 & SMG1 \\
\hline ACBD7 & TRIP12 & PTPMT1 & ASB3 & MTG1 & ANKRD12 \\
\hline STK33 & HEXIM1 & RBM45 & ATG9A & ANKIB1 & B3GALNT2 \\
\hline C12orf43 & SLC25A15 & NDUFAF5 & BAG4 & NOA1 & SFRP2 \\
\hline VPRBP & FOXK1 & GPM6B & POLE & TRADD & AMFR \\
\hline RPS6KA1 & PLA2G4A & SELO & PROM1 & CHTF18 & BOD1 \\
\hline SPC24 & KAT7 & RAB17 & IGFBP6 & PNPLA4 & AGTRAP \\
\hline UBE2Q1 & HIGD2A & RAPH1 & SDF2 & ARHGAP4 & ODR4 \\
\hline MRPS18C & QSOX1 & COX17 & CHUK & RAPGEF2 & GINS1 \\
\hline DFFA & CENPV & PTPN9 & FUT11 & ERMP1 & SOGA1 \\
\hline DHX32 & GEMIN6 & HDHD2 & GLE1 & PTPRZ1 & CREG1 \\
\hline GATC & PDXP & MID1 & WRAP53 & POU2F1 & CA2 \\
\hline APPL1 & TMEM14C & TXNIP & SLC7A3 & FABP6 & ITGA2 \\
\hline CWC22 & MPDZ & PIGG & ACTB & INCENP & CARNMT1 \\
\hline RHBDD2 & MRPL38 & PUM1 & HPDL & NME4 & CDKN2A \\
\hline TRIM27 & ARHGEF10L & BDH1 & CDC26 & CTU1 & ATF7 \\
\hline HMGXB4 & L3MBTL3 & MAP1LC3A & ISLR & URB1 & MRPL21 \\
\hline \multicolumn{6}{|l|}{ Continued } \\
\hline
\end{tabular}


(i) Proteins enriched more than two-fold in c-MYC-WT compared with MYCL-WT (SeV)

\begin{tabular}{|c|c|c|c|c|c|}
\hline CAMK1 & RILPL1 & WDR37 & IGF2BP1 & NAPEPLD & DPH6 \\
\hline FCF1 & ANKRD29 & ANAPC13 & CD3EAP & WDR89 & SLC25A17 \\
\hline DNA2 & CENPM & CEP170B & GCA & CSTF2T & NKRF \\
\hline SLC35A2 & PMS1 & SLC5A6 & COQ5 & SPR & RBM15B \\
\hline USP19 & LAMA4 & DNM1L & INTS2 & BCAS3 & KIF22 \\
\hline NHEJ1 & RPP40 & TNC & SPNS1 & RPRD1A & DDB2 \\
\hline IFIT5 & ARMCX1 & FAT1 & DAP & BMPR1A & NPHP3 \\
\hline ARID3A & MRPL13 & ZBED1 & \begin{tabular}{|l|} 
GTF2H1 \\
\end{tabular} & PATZ1 & $\mathrm{DPH} 2$ \\
\hline C11orf98 & RRP7A & AKAP1 & ANAPC1 & NUDT16 & CD74 \\
\hline DCAKD & ASB6 & DNMBP & UTP11L & TUBG1 & HAUS7 \\
\hline GAA & PDK1 & DOHH & ISYNA1 & BRMS1 & EXOC6 \\
\hline ARAP3 & CHKB & NOL8 & DDX52 & ORC5 & COA7 \\
\hline NAA40 & IQSEC1 & TRIM24 & DNTTIP1 & HEATR6 & SH3BP4 \\
\hline ZMYM3 & MED30 & CLASP2 & PRPF38B & TMEM256 & GTF3C2 \\
\hline MRPL10 & NDUFAF7 & VCPIP1 & HSPB11 & CASP7 & TOR1B \\
\hline ITPR2 & PRPF39 & GCFC2 & KIF21A & DPPA4 & TIMM8B \\
\hline GTF2H4 & MRPL16 & BAZ1A & EXOC6B & MRPL41 & POLR3B \\
\hline USP9Y & SYNE2 & MID1IP1 & MOCS2 & METTL5 & PRUNE \\
\hline UBE2V2 & CDH1 & GJA1 & CHMP6 & RCC1 & RAPGEF6 \\
\hline COMMD9 & HMBS & SQLE & IGFBP2 & DIAPH2 & EPB41 \\
\hline HIST1H1E & MAP3K4 & CDK18 & ARL15 & UQCC1 & HTATIP2 \\
\hline PTDSS2 & TATDN2 & MTA1 & SNX18 & EIF1B & MAEA \\
\hline SCAF1 & UBE2J2 & NUP37 & BAG1 & TSSC4 & RCOR2 \\
\hline FAM213A & MFF & TTLL12 & UBXN6 & EFEMP2 & ZZEF1 \\
\hline NFYB & PDS5B & FXN & AGPAT5 & ARFGEF2 & TCOF1 \\
\hline SCAF11 & RAPGEF1 & PARG & PRIM1 & USF1 & L1TD1 \\
\hline SEPHS1 & BRD8 & POP7 & EXT2 & TSEN34 & CAMKV \\
\hline SLC29A1 & MBD3 & PPIF & MTX3 & COG1 & FASTKD2 \\
\hline ARL14EP & MRPL40 & CD320 & MBD1 & VARS2 & BRD3 \\
\hline MRPS18B & ACY1 & PPIE & RIF1 & VPS8 & POGZ \\
\hline RSL1D1 & PAK1 & STRBP & TERF2 & TOP2A & SLC39A10 \\
\hline QRICH1 & CISD1 & POLB & DHX37 & TRMT5 & TIMMDC1 \\
\hline TRABD & SON & SET & LYST & RNASEH2B & MVK \\
\hline ZC3HC1 & NDUFC1 & HSPA4L & EXOSC1 & TCEANC2 & NASP \\
\hline CYP2S1 & NSMCE3 & HPRT1 & HUS1 & \begin{tabular}{|l|} 
PRRC2A \\
\end{tabular} & RDH13 \\
\hline KANK1 & PHF14 & RBM7 & SIRT5 & QPCTL & CROCC \\
\hline LIMK2 & CWF19L1 & KIF1B & VPS39 & CHD7 & YPEL5 \\
\hline SLC7A5 & VPS25 & LRWD1 & FPGS & NT5C3A & NCKIPSD \\
\hline PSIP1 & CELF2 & MDC1 & ANP32A & ACADSB & GPC4 \\
\hline TUBB4A & BOP1 & NT5C & CTSC & ANKLE2 & ORC4 \\
\hline UTP18 & CHST14 & NUS1 & PLK1 & GPKOW & SIKE1 \\
\hline ADAM15 & NUDCD2 & SSU72 & NFYC & LIG1 & MSH2 \\
\hline HNRNPR & MSI2 & STK26 & EBP & ITPK1 & STAU2 \\
\hline URI1 & SLC7A8 & MANBAL & RNGTT & PRIM2 & SEC24B \\
\hline MRPS34 & RMND5A & F11R & SCAF8 & RPAP1 & SPATA5L1 \\
\hline RCC2 & POLRMT & SERF2 & TMEM115 & PARP1 & C2CD5 \\
\hline BEND3 & BRAT1 & \begin{tabular}{|l|} 
TERF2IP \\
\end{tabular} & MT-ND2 & FAM136A & NOP2 \\
\hline ARL5B & GFM2 & WDR3 & AARSD1 & PPID & HRSP12 \\
\hline CHD2 & MRPL45 & YTHDF1 & THEMIS & INTS4 & PLEKHA1 \\
\hline CBX3 & SIRT7 & CENPF & CAMSAP2 & MCC & PTPN2 \\
\hline NANOG & SLBP & MCAT & MRPL24 & MZT1 & ANAPC4 \\
\hline HIST1H1A & KPNA2 & MED14 & NDUFB9 & NEFL & DUS3L \\
\hline HSP90AA4P & CMSS1 & ZNF706 & PTRHD1 & PBRM1 & ABI3BP \\
\hline MED10 & PFAS & BRIX1 & QRSL1 & \begin{tabular}{|l|} 
THNSL1 \\
\end{tabular} & ADRM1 \\
\hline CECR2 & NCOA5 & ABRACL & WIPF2 & USP28 & GEMIN4 \\
\hline CHD1 & SBNO1 & LSM12 & COG2 & TARS2 & KIF11 \\
\hline
\end{tabular}


(i) Proteins enriched more than two-fold in c-MYC-WT compared with MYCL-WT (SeV)

\begin{tabular}{|c|c|c|c|c|c|}
\hline CHD1L & MLH1 & MNAT1 & DDX47 & SRPK1 & NACAP1 \\
\hline XPC & NELFCD & MPP6 & HAUS6 & TANC1 & PPAN \\
\hline MRPL33 & PUM2 & MRPL15 & FAM65A & ERI3 & TOM1L2 \\
\hline TIMM13 & SPRYD4 & MICU1 & \begin{tabular}{|l|} 
HMGN5 \\
\end{tabular} & XPO4 & WDR43 \\
\hline DDX20 & YTHDC2 & SLC25A22 & CACUL1 & PCF11 & PRC1 \\
\hline DNAAF2 & ACAT1 & RWDD4 & DHPS & PFKM & NUDT16L1 \\
\hline MTMR2 & GPN1 & SMARCA4 & EI24 & SCFD2 & РРР3СВ \\
\hline RNASEH2A & GTF2E2 & SMARCD1 & ADCK3 & TRMT1 & RABEP1 \\
\hline CD97 & GLT8D1 & UBR5 & HLTF & \begin{tabular}{|l|} 
TXNRD2 \\
\end{tabular} & TDP2 \\
\hline $\mathrm{CCNY}$ & NCAPD2 & USP48 & LRCH2 & UBQLN1 & TRIP10 \\
\hline MCM3 & HSPA14 & ZBTB8OS & \begin{tabular}{|l|} 
MAP2K7 \\
\end{tabular} & АРОВЕС3С & TTC27 \\
\hline DYSF & MINA & BAK1 & HERC2 & POLG2 & TRAP1 \\
\hline EXOSC7 & RCL1 & C17orf62 & PUS7 & CASP6 & ISOC1 \\
\hline THYN1 & MYL6 & CBR4 & RFC3 & SRSF10 & LCLAT1 \\
\hline HIBADH & UHRF1 & DHX57 & MCM6 & UBL4A & MRPS17 \\
\hline TFRC & GALK2 & MKI67 & CEBPZ & CHRAC1 & TTC4 \\
\hline METTL3 & GSTZ1 & UBE2O & \begin{tabular}{|l|} 
LRSAM1 \\
\end{tabular} & NSA2 & AGPAT4 \\
\hline MRPS5 & MAP3K7 & ZNF330 & MLLT11 & HK2 & BLMH \\
\hline PCCA & LYPLAL1 & CPSF2 & PEX3 & INPP4A & SORD \\
\hline SLC35F2 & \begin{tabular}{|l|} 
MRPL23 \\
\end{tabular} & GNL3 & WBP11 & SAP30 & INPPL1 \\
\hline TBC1D9B & MRPS31 & GMPS & \begin{tabular}{|l|} 
IMPDH2 \\
\end{tabular} & SSRP1 & LBR \\
\hline TRIM28 & NELFB & LDAH & DDX21 & TWSG1 & RBMX \\
\hline NDUFAF1 & PHF6 & TFB2M & EBNA1BP2 & ZMYM2 & MLYCD \\
\hline UROD & PKP3 & NUBP2 & FANCI & ARIH2 & ZNF22 \\
\hline USP11 & ARFRP1 & PKN1 & FAR1 & MYBBP1A & PDS5A \\
\hline CHMP7 & COASY & SAP18 & GTF3C4 & SMARCAD1 & DNAAF5 \\
\hline CTAGE5 & GART & UBE2I & MRPL27 & ENY2 & PDK3 \\
\hline RUVBL1 & PEAK1 & WDR6 & MYEF2 & PWP2 & RPA2 \\
\hline EXOSC4 & FRA10AC1 & ADAD2 & NARS2 & FAM64A & XRCC4 \\
\hline GTF2E1 & DDX51 & BMS1 & OGFR & MRPL3 & CHAC2 \\
\hline PES1 & \begin{tabular}{|l|} 
IGF2BP3 \\
\end{tabular} & LDHB & ORC3 & MSH6 & DPYSL5 \\
\hline HEATR1 & \begin{tabular}{|l|} 
KATNA1 \\
\end{tabular} & PTMA & \begin{tabular}{|l|} 
SLC52A2 \\
\end{tabular} & NLE1 & MCM7 \\
\hline POLD1 & MCM2 & CXorf56 & SMAD5 & GPATCH4 & \begin{tabular}{|l|} 
STOML2 \\
\end{tabular} \\
\hline FLVCR1 & MYH14 & DAXX & ZCCHC8 & TAMM41 & TOMM34 \\
\hline LGALS3BP & PARN & KIF1BP & C5orf22 & TP53RK & NACC1 \\
\hline MRPS30 & TKT & ELAC2 & PRKCI & PDCD11 & DNAJB4 \\
\hline MTMR12 & SUPV3L1 & DARS2 & RAVER1 & SIGMAR1 & DHODH \\
\hline SLC3A2 & BTAF1 & HSDL1 & EIF3C & TBPL1 & NOC2L \\
\hline MRPS28 & CADM1 & MRPL37 & SRI & BZW2 & NDUFC2 \\
\hline РCCB & DNTTIP2 & PCBD2 & TRIM2 & CPSF1 & RABGGTB \\
\hline RFC5 & ECT2 & AGTPBP1 & TRIM22 & ECM29 & PANK4 \\
\hline C11orf73 & MCAM & THUMPD1 & WDR18 & RNPS1 & SCO1 \\
\hline TIMM17B & \begin{tabular}{|l|} 
INTS8 \\
\end{tabular} & TUSC3 & DCAF16 & GTF2A1 & SIRT1 \\
\hline UTP6 & MRPL11 & XRN1 & DCTPP1 & HSPBP1 & TUFM \\
\hline WDR92 & PIR & ACAA2 & \begin{tabular}{|l|} 
DNAJC2 \\
\end{tabular} & NPM3 & ADNP \\
\hline CERS6 & \begin{tabular}{|l|} 
NUDT12 \\
\end{tabular} & APTX & DSG2 & NTHL1 & BOLA3 \\
\hline DDX24 & NUP35 & ATL2 & NOL11 & DDX31 & ATPAF1 \\
\hline $\mathrm{GCDH}$ & RCHY1 & C12orf10 & HIP1R & PRPS2 & MCM5 \\
\hline WDR75 & RHOT1 & KIAA1211 & DDX54 & RSPRY1 & CDC123 \\
\hline NOP16 & TACO1 & GLMN & HOOK1 & WNK1 & NUP155 \\
\hline POLR1A & TBL3 & GNAI1 & KIAA2013 & C7orf50 & GUSB \\
\hline GUF1 & TIMM17A & GRWD1 & LBH & CCNK & ILKAP \\
\hline SMYD5 & AGL & POR & LIG3 & CMTR1 & LRBA \\
\hline WDR5 & TOMM5 & ARL8A & PCK2 & PDCD4 & NAT10 \\
\hline ACO1 & TSC2 & GNL3L & MTFP1 & ABT1 & KEAP1 \\
\hline ANKRD28 & UBTF & STK3 & SCAP & PPAT & PSAT1 \\
\hline
\end{tabular}




\begin{tabular}{|c|c|c|c|c|c|}
\hline \multicolumn{6}{|c|}{ (i) Proteins enriched more than two-fold in c-MYC-WT compared with MYCL-WT (SeV) } \\
\hline APOA1BP & WDR54 & SYT1 & SCRIB & РTBP3 & RNASEH2C \\
\hline APOO & ASF1A & GSPT2 & SDHB & SUPT16H & DBR1 \\
\hline PSME3 & PPWD1 & UBQLN4 & CHAF1A & TRMT10C & SACS \\
\hline BYSL & $\mathrm{CDH} 13$ & USP24 & ARID1A & GJB2 & TEX10 \\
\hline UBA2 & CLUH & ARL2 & AS3MT & ABCC1 & MRPL57 \\
\hline DDX56 & EIF2D & ATP1B3 & DCAF8 & AGK & AIF1L \\
\hline RBM42 & FEN1 & CCDC12 & \begin{tabular}{|l|} 
ELOVL6 \\
\end{tabular} & EFNB1 & NCAPG2 \\
\hline SARS2 & OSBPL2 & CDCA8 & GNA13 & ATF7IP & CCDC50 \\
\hline ADI1 & HAUS8 & Clorf131 & GOLM1 & DEK & DTD1 \\
\hline MCL1 & INTS1 & AHCY & GYS1 & PAICS & DTWD1 \\
\hline CCDC59 & MAK16 & RUVBL2 & ISY1 & ECT2L & GTPBP10 \\
\hline ZNF593 & RBM26 & FDXR & MRI1 & HS1BP3 & LSM6 \\
\hline DNAJC8 & SALL4 & AATF & LETM1 & LAS1L & MRPS11 \\
\hline SMARCA5 & TMPO & LTA4H & MYCBP & MRPL19 & NEPRO \\
\hline РCBP2 & WBSCR22 & $\mathrm{CCNH}$ & DLGAP5 & NDUFS7 & NOP58 \\
\hline HDAC2 & WDR48 & GLTSCR2 & PELP1 & HARS2 & NT5DC1 \\
\hline HIST1H1B & TOX4 & TPI1 & LUC7L & ATP11C & NXF1 \\
\hline HNRNPC & VRK1 & KDM2A & COMMD8 & CACNA2D1 & RAD21 \\
\hline HNRNPU & WIPI1 & USP39 & SSB & CDCA7L & SNF8 \\
\hline LARS & ASH2L & VPS36 & ZNF346 & CRNKL1 & TELO2 \\
\hline LRRC57 & AASDHPPT & CNPY3 & GLUL & CWC27 & TNPO3 \\
\hline CHORDC1 & CKAP2 & CXADR & ILF2 & PEG10 & TTI2 \\
\hline MANBA & DCAF13 & PLS1 & LVRN & C1QBP & UBE2A \\
\hline MEMO1 & EIF4A3 & EXTL2 & MED24 & GLYR1 & UBXN1 \\
\hline MRPS18A & EIF5B & NIFK & POLR2H & HMOX2 & ABCB6 \\
\hline NDUFB4 & FNBP1L & NOL10 & KIF5C & EXOSC9 & ATP2B1 \\
\hline TOMM40 & RBM28 & GSTP1 & TATDN1 & FTSJ3 & DAGLB \\
\hline PLCG1 & IFI16 & HSPA4 & TMEM192 & MPI & SMPD4 \\
\hline POLE4 & KDM1A & \begin{tabular}{|l|} 
NUP188 \\
\end{tabular} & TSFM & DLAT & FAM210A \\
\hline POLR2D & NOL6 & PRPF40A & UBAP2 & MSTO1 & GTF2I \\
\hline SHPK & NUP133 & RBM12B & DAB2IP & SAFB & LARP1 \\
\hline SPCS1 & PDHA1 & STX18 & PPFIA1 & NUP50 & METTL13 \\
\hline SAAL1 & QTRTD1 & SUPT6H & ADSL & QSER1 & POLR3C \\
\hline TRMT1L & ASUN & BMP1 & AFG3L2 & GEMIN5 & NEU1 \\
\hline PEX6 & HDAC3 & NDC80 & MRRF & HMGN1 & TIMM44 \\
\hline REEP6 & OTX2 & LPCAT1 & MTPAP & SNU13 & UMPS \\
\hline TRIAP1 & TUT1 & GRSF1 & NAA20 & ACSS3 & NCK1 \\
\hline ERBB2 & CCDC28B & XRN2 & NDUFAF4 & MIF & PPP2R5A \\
\hline SGSH & CRADD & MT-ND4 & GATAD2A & MRPL9 & RAD50 \\
\hline OSGEPL1 & ADGRL2 & MTUS2 & NRBF2 & FAM192A & DNPEP \\
\hline THAP11 & HEATR5B & RABGEF1 & POP1 & HEATR3 & SRRM2 \\
\hline CACTIN & PRKD1 & MRPL50 & PRMT1 & NIPBL & STK4 \\
\hline SLC25A35 & ECE2 & RPL13A & SDCBP & NTMT1 & TBRG4 \\
\hline ZFAND6 & DSEL & SDAD1 & RPL21 & PDCD5 & PPIG \\
\hline RALGAPB & MYO5C & TRMT6 & RBM39 & GULP1 & DCP1A \\
\hline IGSF1 & POLG & UBE3A & SLC30A1 & RFC4 & TUBAL3 \\
\hline MGRN1 & TPK1 & KCTD10 & SMAP & ACIN1 & UBXN7 \\
\hline TMEM41A & DNAL1 & F8A1,F8A2,F8A3 & TKFC & BSG & ARHGEF40 \\
\hline FBP1 & FBXL6 & DFFB & TRIM33 & EHBP1 & ATP5S \\
\hline CDC20 & PLEKHA7 & WDR73 & CD70 & EMC3 & SLC9A3R1 \\
\hline COBLL1 & TRIM9 & SP1 & YARS2 & RBM19 & CKMT1A,CKMT1B \\
\hline HSP90AB3P & MRPL35 & CCNA2 & HDDC2 & KDM3B & PPA1 \\
\hline SMARCAL1 & MALSU1 & AURKB & PPM1G & EARS2 & HSP90AA1 \\
\hline SH3GL3 & MPHOSPH6 & NACC2 & HMGB2 & GTPBP4 & ABCB10 \\
\hline CYP2U1 & BSDC1 & TLK1 & LEO1 & MFAP1 & GIT1 \\
\hline FLCN & TYRO3 & SH3PXD2A & EMC4 & CPS1 & INTS9 \\
\hline
\end{tabular}


(i) Proteins enriched more than two-fold in c-MYC-WT compared with MYCL-WT (SeV)

\begin{tabular}{|c|c|c|c|c|c|}
\hline IRS2 & SIRT3 & FUT8 & CDK2 & TNFAIP6 & SDHAF4 \\
\hline KITLG & ALDH3A1 & GPR180 & CFAP36 & ANK3 & CBX2 \\
\hline RBBP9 & EML2 & POLR3GL & FN3K & VWA5A & RAVER2 \\
\hline ATXN7L3B & \begin{tabular}{|l|} 
TIMM21 \\
\end{tabular} & SLC2A3 & CA14 & CRLF3 & SYT6 \\
\hline TMEM161A & MTM1 & METTL15 & NKAP & CDS2 & MRS2 \\
\hline MARS2 & ERCC2 & TDP1 & MFAP4 & ANAPC16 & CARS2 \\
\hline NOLC1 & IGHMBP2 & MRPL34 & FECH & PARP2 & ING1 \\
\hline ADNP2 & STEAP3 & AK6 & PDZD8 & EPB41L5 & PEX16 \\
\hline ZER1 & CKS1B & GGPS1 & DBNDD1 & MIEF1 & FUCA1 \\
\hline ADSSL1 & POTEJ & TMEM209 & CCNL2 & TOP3A & ULK1 \\
\hline MGA & FAM162A & AMMECR1 & ISG20L2 & CEP78 & NOM1 \\
\hline PAPD4 & PROCR & IFRD2 & LRRC41 & UBR3 & PHF3 \\
\hline RIN1 & SPPL2B & ARAF & DNM2 & HPS5 & PSEN1 \\
\hline PARD3 & ARHGEF16 & RHPN2 & PRPF18 & SEMA4C & RPUSD3 \\
\hline NYNRIN & ARHGEF7 & VRTN & PHF10 & DMD & RPL26L1 \\
\hline RANBP6 & CNOT4 & TSPYL5 & CDC25C & REEP4 & FADD \\
\hline INPP5F & ZBTB7A & GPN3 & \begin{tabular}{|l|} 
RBPMS2 \\
\end{tabular} & BRAF & ORC6 \\
\hline CACNA2D2 & AP1B1 & NCAPD3 & RRP8 & MASTL & POLR2M \\
\hline CASC3 & NCL & Clorf174 & LRRC14 & SLC27A3 & ACSF3 \\
\hline DHRS11 & RBM23 & WDR55 & CAMK4 & NDRG3 & ALS2 \\
\hline NOVA1 & SOX3 & CLCN7 & EHMT1 & C7orf26 & NSUN5 \\
\hline NMRAL1 & STK25 & NFKB2 & OSBPL1A & VPS37B & RAD23A \\
\hline HS2ST1 & LYAR & PHKA1 & SDC4 & MGST2 & SNTB1 \\
\hline MEN1 & WDR4 & DDX28 & C1orf198 & AKAP9 & COQ9 \\
\hline STYX & PHF5A & PCDH1 & TMSB4X & AP1G2 & MYO1G \\
\hline UCKL1 & APC & TBC1D15 & FASTKD1 & APCDD1L & MARH5 \\
\hline ULK3 & LONP2 & SETD1A & ETFDH & ANKS1A & LRP8 \\
\hline PALD1 & ANAPC5 & CARMIL1 & GATM & PANX1 & NME3 \\
\hline UBA52 & ZNF806 & NCOR2 & DVL2 & CTDP1 & PHKB \\
\hline GINS3 & DNPH1 & CDCA5 & BCKDK & TTF1 & TGFBRAP1 \\
\hline HAUS2 & \begin{tabular}{|l|} 
HMGCR \\
\end{tabular} & SNCA & KLHDC4 & TBP & AP1M2 \\
\hline E2F4 & AFAP1L1 & ZMYM6NB & N4BP2 & TRIT1 & CCDC134 \\
\hline ATL1 & \begin{tabular}{|l|} 
INTS6 \\
\end{tabular} & CHD8 & SPINT2 & RASA2 & NCK2 \\
\hline MAL2 & ATAD3A & SLC25A32 & LSAMP & ACOT8 & KIFAP3 \\
\hline JARID2 & \begin{tabular}{|l|} 
CLSTN1 \\
\end{tabular} & USP36 & PTGIS & PIAS4 & TMEM41B \\
\hline SEC14L1 & TUBGCP4 & GEMIN8 & VWA9 & RPP25L & NRBP2 \\
\hline DOLPP1 & WARS2 & PLEKHA6 & MRGBP & ZCCHC6 & ZFP36L1 \\
\hline SLC4A7 & SCARB1 & ARID1B & PMF1 & XXYLT1 & ANKRD50 \\
\hline MT-CO1 & MET & RBM47 & LIN28B & EXD2 & GORAB \\
\hline GCSH & PLTP & PRKAB1 & CUTC & SDSL & FARS2 \\
\hline LRRC8E & ARHGAP12 & FBXW9 & PMS2 & NAA30 & STRA13 \\
\hline FASTKD5 & ZCCHC10 & TTK & BNC2 & COX16 & BCS1L \\
\hline NDE1 & STX3 & LARP1B & PTCD1 & TPD52 & SMG1 \\
\hline ACBD7 & TRIP12 & PTPMT1 & ASB3 & MTG1 & ANKRD12 \\
\hline STK33 & \begin{tabular}{|l|} 
HEXIM1 \\
\end{tabular} & RBM45 & ATG9A & ANKIB1 & B3GALNT2 \\
\hline C12orf43 & SLC25A15 & NDUFAF5 & BAG4 & NOA1 & SFRP2 \\
\hline VPRBP & FOXK1 & GPM6B & POLE & TRADD & AMFR \\
\hline RPS6KA1 & PLA2G4A & SELO & PROM1 & CHTF18 & BOD1 \\
\hline SPC24 & KAT7 & RAB17 & IGFBP6 & PNPLA4 & AGTRAP \\
\hline UBE2Q1 & HIGD2A & RAPH1 & SDF2 & ARHGAP4 & ODR4 \\
\hline MRPS18C & QSOX1 & COX17 & CHUK & RAPGEF2 & GINS1 \\
\hline DFFA & CENPV & PTPN9 & FUT11 & ERMP1 & SOGA1 \\
\hline DHX32 & \begin{tabular}{|l|} 
GEMIN6 \\
\end{tabular} & HDHD2 & GLE1 & PTPRZ1 & CREG1 \\
\hline GATC & PDXP & MID1 & WRAP53 & POU2F1 & CA2 \\
\hline APPL1 & TMEM14C & TXNIP & SLC7A3 & FABP6 & ITGA2 \\
\hline CWC22 & MPDZ & PIGG & ACTB & INCENP & CARNMT1 \\
\hline
\end{tabular}


(i) Proteins enriched more than two-fold in c-MYC-WT compared with MYCL-WT (SeV)

\begin{tabular}{|c|c|c|c|c|c|}
\hline RHBDD2 & MRPL38 & PUM1 & HPDL & NME4 & CDKN2A \\
\hline TRIM27 & ARHGEF10L & BDH1 & CDC26 & CTU1 & ATF7 \\
\hline HMGXB4 & L3MBTL3 & MAP1LC3A & ISLR & URB1 & MRPL21 \\
\hline CAMK1 & RILPL1 & WDR37 & \begin{tabular}{|l|} 
IGF2BP1 \\
\end{tabular} & NAPEPLD & DPH6 \\
\hline FCF1 & ANKRD29 & ANAPC13 & CD3EAP & WDR89 & SLC25A17 \\
\hline DNA2 & CENPM & CEP170B & GCA & CSTF2T & NKRF \\
\hline SLC35A2 & PMS1 & SLC5A6 & COQ5 & SPR & RBM15B \\
\hline USP19 & LAMA4 & DNM1L & \begin{tabular}{|l|} 
INTS2 \\
\end{tabular} & BCAS3 & KIF22 \\
\hline NHEJ1 & RPP40 & TNC & SPNS1 & RPRD1A & DDB2 \\
\hline IFIT5 & ARMCX1 & FAT1 & DAP & BMPR1A & NPHP3 \\
\hline ARID3A & MRPL13 & ZBED1 & GTF2H1 & PATZ1 & DPH2 \\
\hline C11orf98 & RRP7A & AKAP1 & ANAPC1 & NUDT16 & CD74 \\
\hline DCAKD & ASB6 & DNMBP & UTP11L & TUBG1 & HAUS7 \\
\hline GAA & PDK1 & DOHH & ISYNA1 & BRMS1 & EXOC6 \\
\hline ARAP3 & CHKB & NOL8 & DDX52 & ORC5 & COA7 \\
\hline NAA40 & IQSEC1 & TRIM24 & DNTTIP1 & HEATR6 & SH3BP4 \\
\hline ZMYM3 & MED30 & CLASP2 & PRPF38B & TMEM256 & GTF3C2 \\
\hline MRPL10 & NDUFAF7 & VCPIP1 & HSPB11 & CASP7 & TOR1B \\
\hline ITPR2 & PRPF39 & GCFC2 & KIF21A & DPPA4 & TIMM8B \\
\hline GTF2H4 & MRPL16 & BAZ1A & \begin{tabular}{|l|} 
EXOC6B \\
\end{tabular} & MRPL41 & POLR3B \\
\hline USP9Y & SYNE2 & MID1IP1 & MOCS2 & METTL5 & PRUNE \\
\hline UBE2V2 & $\mathrm{CDH1}$ & GJA1 & CHMP6 & RCC1 & RAPGEF6 \\
\hline COMMD9 & HMBS & SQLE & IGFBP2 & DIAPH2 & EPB41 \\
\hline HIST1H1E & MAP3K4 & CDK18 & ARL15 & UQCC1 & HTATIP2 \\
\hline PTDSS2 & TATDN2 & MTA1 & SNX18 & EIF1B & MAEA \\
\hline SCAF1 & UBE2J2 & NUP37 & BAG1 & TSSC4 & RCOR2 \\
\hline FAM213A & MFF & TTLL12 & UBXN6 & EFEMP2 & ZZEF1 \\
\hline NFYB & PDS5B & FXN & AGPAT5 & ARFGEF2 & TCOF1 \\
\hline SCAF11 & RAPGEF1 & PARG & PRIM1 & USF1 & L1TD1 \\
\hline SEPHS1 & BRD8 & POP7 & EXT2 & TSEN34 & CAMKV \\
\hline SLC29A1 & MBD3 & PPIF & MTX3 & COG1 & FASTKD2 \\
\hline ARL14EP & MRPL40 & CD320 & MBD1 & VARS2 & BRD3 \\
\hline MRPS18B & ACY1 & PPIE & RIF1 & VPS8 & POGZ \\
\hline RSL1D1 & PAK1 & STRBP & TERF2 & TOP2A & SLC39A10 \\
\hline QRICH1 & CISD1 & POLB & DHX37 & TRMT5 & TIMMDC1 \\
\hline TRABD & SON & SET & LYST & RNASEH2B & MVK \\
\hline ZC3HC1 & NDUFC1 & HSPA4L & EXOSC1 & TCEANC2 & NASP \\
\hline CYP2S1 & NSMCE3 & HPRT1 & HUS1 & PRRC2A & RDH13 \\
\hline KANK1 & PHF14 & RBM7 & SIRT5 & QPCTL & CROCC \\
\hline LIMK2 & CWF19L1 & KIF1B & VPS39 & CHD7 & YPEL5 \\
\hline SLC7A5 & VPS25 & LRWD1 & FPGS & NT5C3A & NCKIPSD \\
\hline PSIP1 & CELF2 & MDC1 & ANP32A & ACADSB & GPC4 \\
\hline TUBB4A & BOP1 & NT5C & CTSC & ANKLE2 & ORC4 \\
\hline UTP18 & CHST14 & NUS1 & PLK1 & GPKOW & SIKE1 \\
\hline ADAM15 & NUDCD2 & SSU72 & NFYC & LIG1 & MSH2 \\
\hline HNRNPR & MSI2 & STK26 & EBP & ITPK1 & STAU2 \\
\hline URI1 & SLC7A8 & MANBAL & RNGTT & PRIM2 & SEC24B \\
\hline MRPS34 & RMND5A & F11R & SCAF8 & RPAP1 & SPATA5L1 \\
\hline RCC2 & POLRMT & SERF2 & TMEM115 & PARP1 & C2CD5 \\
\hline BEND3 & BRAT1 & TERF2IP & \begin{tabular}{|l|} 
MT-ND2 \\
\end{tabular} & FAM136A & NOP2 \\
\hline ARL5B & GFM2 & WDR3 & AARSD1 & PPID & HRSP12 \\
\hline CHD2 & MRPL45 & YTHDF1 & \begin{tabular}{|l|} 
THEMIS \\
\end{tabular} & INTS4 & PLEKHA1 \\
\hline CBX3 & SIRT7 & CENPF & CAMSAP2 & MCC & PTPN2 \\
\hline NANOG & SLBP & MCAT & MRPL24 & MZT1 & ANAPC4 \\
\hline HIST1H1A & KPNA2 & MED14 & \begin{tabular}{|l|} 
NDUFB9 \\
\end{tabular} & NEFL & DUS3L \\
\hline HSP90AA4P & CMSS1 & ZNF706 & PTRHD1 & PBRM1 & ABI3BP \\
\hline \multicolumn{6}{|l|}{ Continued } \\
\hline
\end{tabular}


(i) Proteins enriched more than two-fold in c-MYC-WT compared with MYCL-WT (SeV)

\begin{tabular}{|c|c|c|c|c|c|}
\hline MED10 & PFAS & BRIX1 & QRSL1 & THNSL1 & ADRM1 \\
\hline CECR2 & NCOA5 & ABRACL & WIPF2 & USP28 & GEMIN4 \\
\hline CHD1 & SBNO1 & LSM12 & COG2 & TARS2 & KIF11 \\
\hline CHD1L & MLH1 & MNAT1 & DDX47 & SRPK1 & NACAP1 \\
\hline XPC & NELFCD & MPP6 & HAUS6 & TANC1 & PPAN \\
\hline MRPL33 & PUM2 & MRPL15 & FAM65A & ERI3 & TOM1L2 \\
\hline TIMM13 & \begin{tabular}{|l|} 
SPRYD4 \\
\end{tabular} & MICU1 & HMGN5 & XPO4 & WDR43 \\
\hline DDX20 & YTHDC2 & SLC25A22 & CACUL1 & PCF11 & PRC1 \\
\hline DNAAF2 & ACAT1 & RWDD4 & DHPS & PFKM & NUDT16L1 \\
\hline MTMR2 & GPN1 & SMARCA4 & EI24 & SCFD2 & РРР3СВ \\
\hline RNASEH2A & GTF2E2 & SMARCD1 & ADCK3 & TRMT1 & RABEP1 \\
\hline CD97 & \begin{tabular}{|l|} 
GLT8D1 \\
\end{tabular} & UBR5 & HLTF & \begin{tabular}{|l|} 
TXNRD2 \\
\end{tabular} & TDP2 \\
\hline CCNY & NCAPD2 & USP48 & LRCH2 & UBQLN1 & TRIP10 \\
\hline MCM3 & HSPA14 & ZBTB8OS & \begin{tabular}{|l|} 
MAP2K7 \\
\end{tabular} & AРОВЕС $3 \mathrm{C}$ & TTC27 \\
\hline DYSF & MINA & BAK1 & HERC2 & POLG2 & TRAP1 \\
\hline EXOSC7 & RCL1 & \begin{tabular}{|l|} 
C17orf62 \\
\end{tabular} & PUS7 & CASP6 & ISOC1 \\
\hline THYN1 & MYL6 & CBR4 & RFC3 & SRSF10 & LCLAT1 \\
\hline HIBADH & UHRF1 & DHX57 & MCM6 & UBL4A & MRPS17 \\
\hline TFRC & GALK2 & MKI67 & CEBPZ & CHRAC1 & TTC4 \\
\hline METTL3 & GSTZ1 & UBE2O & \begin{tabular}{|l|} 
LRSAM1 \\
\end{tabular} & NSA2 & AGPAT4 \\
\hline MRPS5 & MAP3K7 & ZNF330 & MLLT11 & HK2 & BLMH \\
\hline PCCA & \begin{tabular}{|l|} 
LYPLAL1 \\
\end{tabular} & CPSF2 & PEX3 & INPP4A & SORD \\
\hline SLC35F2 & \begin{tabular}{|l|} 
MRPL23 \\
\end{tabular} & GNL3 & WBP11 & SAP30 & INPPL1 \\
\hline TBC1D9B & MRPS31 & GMPS & \begin{tabular}{|l|} 
IMPDH2 \\
\end{tabular} & SSRP1 & LBR \\
\hline TRIM28 & NELFB & LDAH & DDX21 & TWSG1 & RBMX \\
\hline NDUFAF1 & PHF6 & TFB2M & EBNA1BP2 & ZMYM2 & MLYCD \\
\hline UROD & PKP3 & NUBP2 & FANCI & ARIH2 & ZNF22 \\
\hline USP11 & ARFRP1 & PKN1 & FAR1 & MYBBP1A & PDS5A \\
\hline CHMP7 & COASY & SAP18 & GTF3C4 & SMARCAD1 & DNAAF5 \\
\hline CTAGE5 & GART & UBE2I & \begin{tabular}{|l|} 
MRPL27 \\
\end{tabular} & ENY2 & PDK3 \\
\hline RUVBL1 & PEAK1 & WDR6 & MYEF2 & PWP2 & RPA2 \\
\hline EXOSC4 & FRA10AC1 & ADAD2 & NARS2 & FAM64A & XRCC4 \\
\hline GTF2E1 & DDX51 & BMS1 & OGFR & MRPL3 & CHAC2 \\
\hline PES1 & \begin{tabular}{|l|} 
IGF2BP3 \\
\end{tabular} & LDHB & ORC3 & MSH6 & DPYSL5 \\
\hline HEATR1 & \begin{tabular}{|l|} 
KATNA1 \\
\end{tabular} & PTMA & SLC52A2 & NLE1 & MCM7 \\
\hline POLD1 & MCM2 & CXorf56 & SMAD5 & GPATCH4 & STOML2 \\
\hline FLVCR1 & MYH14 & DAXX & ZCCHC8 & TAMM41 & TOMM34 \\
\hline LGALS3BP & PARN & KIF1BP & C5orf22 & TP53RK & NACC1 \\
\hline MRPS30 & TKT & ELAC2 & PRKCI & PDCD11 & DNAJB4 \\
\hline MTMR12 & SUPV3L1 & DARS2 & RAVER1 & SIGMAR1 & DHODH \\
\hline SLC3A2 & BTAF1 & HSDL1 & EIF3C & TBPL1 & NOC2L \\
\hline MRPS28 & CADM1 & MRPL37 & SRI & BZW2 & NDUFC2 \\
\hline РСCB & DNTTIP2 & PCBD2 & TRIM2 & CPSF1 & RABGGTB \\
\hline RFC5 & ECT2 & AGTPBP1 & TRIM22 & ECM29 & PANK4 \\
\hline C11orf73 & MCAM & THUMPD1 & WDR18 & RNPS1 & SCO1 \\
\hline TIMM17B & \begin{tabular}{|l|} 
INTS8 \\
\end{tabular} & TUSC3 & DCAF16 & GTF2A1 & SIRT1 \\
\hline UTP6 & MRPL11 & XRN1 & \begin{tabular}{|l|} 
DCTPP1 \\
\end{tabular} & HSPBP1 & TUFM \\
\hline WDR92 & PIR & ACAA2 & \begin{tabular}{|l|} 
DNAJC2 \\
\end{tabular} & NPM3 & ADNP \\
\hline CERS6 & \begin{tabular}{|l|} 
NUDT12 \\
\end{tabular} & APTX & DSG2 & NTHL1 & BOLA3 \\
\hline DDX24 & NUP35 & ATL2 & NOL11 & DDX31 & ATPAF1 \\
\hline GCDH & RCHY1 & C12orf10 & HIP1R & PRPS2 & MCM5 \\
\hline WDR75 & RHOT1 & KIAA1211 & DDX54 & RSPRY1 & CDC123 \\
\hline NOP16 & TACO1 & GLMN & HOOK1 & WNK1 & NUP155 \\
\hline POLR1A & TBL3 & GNAI1 & KIAA2013 & C7orf50 & GUSB \\
\hline GUF1 & TIMM17A & GRWD1 & LBH & CCNK & ILKAP \\
\hline SMYD5 & AGL & POR & LIG3 & CMTR1 & LRBA \\
\hline \multicolumn{6}{|l|}{ Continued } \\
\hline
\end{tabular}


(i) Proteins enriched more than two-fold in c-MYC-WT compared with MYCL-WT (SeV)

\begin{tabular}{|c|c|c|c|c|c|}
\hline WDR5 & TOMM5 & ARL8A & PCK2 & PDCD4 & NAT10 \\
\hline ACO1 & TSC2 & GNL3L & MTFP1 & ABT1 & KEAP1 \\
\hline ANKRD28 & UBTF & STK3 & SCAP & PPAT & PSAT1 \\
\hline APOA1BP & WDR54 & SYT1 & SCRIB & PTBP3 & RNASEH2C \\
\hline APOO & ASF1A & GSPT2 & SDHB & SUPT16H & DBR1 \\
\hline PSME3 & PPWD1 & UBQLN4 & CHAF1A & TRMT10C & SACS \\
\hline BYSL & CDH13 & USP24 & ARID1A & GJB2 & TEX10 \\
\hline UBA2 & CLUH & ARL2 & AS3MT & ABCC1 & MRPL57 \\
\hline DDX56 & EIF2D & ATP1B3 & DCAF8 & AGK & AIF1L \\
\hline RBM42 & FEN1 & CCDC12 & \begin{tabular}{|l|} 
ELOVL6 \\
\end{tabular} & EFNB1 & NCAPG2 \\
\hline SARS2 & OSBPL2 & CDCA8 & GNA13 & ATF7IP & CCDC50 \\
\hline ADI1 & HAUS8 & Clorf131 & GOLM1 & DEK & DTD1 \\
\hline MCL1 & INTS1 & AHCY & GYS1 & PAICS & DTWD1 \\
\hline CCDC59 & MAK16 & RUVBL2 & ISY1 & ECT2L & GTPBP10 \\
\hline ZNF593 & RBM26 & FDXR & MRI1 & HS1BP3 & LSM6 \\
\hline DNAJC8 & SALL4 & AATF & LETM1 & LAS1L & MRPS11 \\
\hline SMARCA5 & TMPO & LTA4H & MYCBP & MRPL19 & NEPRO \\
\hline РCBP2 & WBSCR22 & $\mathrm{CCNH}$ & DLGAP5 & NDUFS7 & NOP58 \\
\hline HDAC2 & WDR48 & GLTSCR2 & PELP1 & HARS2 & NT5DC1 \\
\hline HIST1H1B & TOX4 & TPI1 & LUC7L & ATP11C & NXF1 \\
\hline HNRNPC & VRK1 & KDM2A & COMMD8 & CACNA2D1 & RAD21 \\
\hline HNRNPU & WIPI1 & USP39 & SSB & CDCA7L & SNF8 \\
\hline LARS & ASH2L & VPS36 & ZNF346 & CRNKL1 & TELO2 \\
\hline LRRC57 & AASDHPPT & CNPY3 & GLUL & CWC27 & TNPO3 \\
\hline CHORDC1 & CKAP2 & CXADR & ILF2 & PEG10 & TTI2 \\
\hline MANBA & DCAF13 & PLS1 & LVRN & C1QBP & UBE2A \\
\hline MEMO1 & EIF4A3 & EXTL2 & MED24 & GLYR1 & UBXN1 \\
\hline MRPS18A & EIF5B & NIFK & POLR2H & HMOX2 & ABCB6 \\
\hline NDUFB4 & FNBP1L & NOL10 & KIF5C & EXOSC9 & ATP2B1 \\
\hline TOMM40 & RBM28 & GSTP1 & \begin{tabular}{|l|} 
TATDN1 \\
\end{tabular} & FTSJ3 & DAGLB \\
\hline PLCG1 & IFI16 & HSPA4 & TMEM192 & MPI & SMPD4 \\
\hline POLE4 & KDM1A & NUP188 & TSFM & DLAT & FAM210A \\
\hline POLR2D & NOL6 & PRPF40A & UBAP2 & MSTO1 & GTF2I \\
\hline SHPK & NUP133 & RBM12B & DAB2IP & SAFB & LARP1 \\
\hline SPCS1 & PDHA1 & STX18 & PPFIA1 & NUP50 & METTL13 \\
\hline SAAL1 & QTRTD1 & SUPT6H & ADSL & QSER1 & POLR3C \\
\hline TRMT1L & ASUN & BMP1 & AFG3L2 & GEMIN5 & NEU1 \\
\hline PEX6 & HDAC3 & NDC80 & MRRF & HMGN1 & TIMM44 \\
\hline REEP6 & OTX2 & LPCAT1 & MTPAP & SNU13 & UMPS \\
\hline TRIAP1 & TUT1 & GRSF1 & NAA20 & ACSS3 & NCK1 \\
\hline ERBB2 & CCDC28B & XRN2 & NDUFAF4 & MIF & PPP2R5A \\
\hline SGSH & CRADD & MT-ND4 & GATAD2A & MRPL9 & RAD50 \\
\hline OSGEPL1 & ADGRL2 & MTUS2 & NRBF2 & FAM192A & DNPEP \\
\hline THAP11 & HEATR5B & RABGEF1 & POP1 & HEATR3 & SRRM2 \\
\hline CACTIN & PRKD1 & MRPL50 & PRMT1 & NIPBL & STK4 \\
\hline SLC25A35 & ECE2 & RPL13A & SDCBP & NTMT1 & TBRG4 \\
\hline ZFAND6 & DSEL & SDAD1 & RPL21 & PDCD5 & PPIG \\
\hline RALGAPB & MYO5C & TRMT6 & RBM39 & GULP1 & DCP1A \\
\hline IGSF1 & POLG & UBE3A & SLC30A1 & RFC4 & TUBAL3 \\
\hline MGRN1 & TPK1 & KCTD10 & SMAP & ACIN1 & UBXN7 \\
\hline TMEM41A & DNAL1 & F8A1,F8A2,F8A3 & TKFC & BSG & ARHGEF40 \\
\hline FBP1 & FBXL6 & DFFB & TRIM33 & EHBP1 & ATP5S \\
\hline CDC20 & PLEKHA7 & WDR73 & CD70 & EMC3 & SLC9A3R1 \\
\hline COBLL1 & TRIM9 & SP1 & YARS2 & RBM19 & CKMT1A,CKMT1B \\
\hline HSP90AB3P & MRPL35 & CCNA2 & HDDC2 & KDM3B & PPA1 \\
\hline SMARCAL1 & MALSU1 & AURKB & PPM1G & EARS2 & HSP90AA1 \\
\hline
\end{tabular}




\begin{tabular}{|l|l|l|l|l|l|}
\hline \multicolumn{6}{|l|}{ (i) Proteins enriched more than two-fold in c-MYC-WT compared with MYCL-WT (SeV) } \\
\hline SH3GL3 & MPHOSPH6 & NACC2 & HMGB2 & GTPBP4 & ABCB10 \\
\hline CYP2U1 & BSDC1 & TLK1 & LEO1 & MFAP1 & GIT1 \\
\hline FLCN & TYRO3 & SH3PXD2A & EMC4 & CPS1 & INTS9 \\
\hline IRS2 & SIRT3 & FUT8 & CDK2 & TNFAIP6 & SDHAF4 \\
\hline KITLG & ALDH3A1 & GPR180 & CFAP36 & ANK3 & CBX2 \\
\hline RBBP9 & EML2 & POLR3GL & FN3K & VWA5A & RAVER2 \\
\hline
\end{tabular}

(ii) Proteins enriched more than two-fold in c-MYC-WT compared with MYCL-WT (EpiP)

\begin{tabular}{|c|c|c|c|c|c|}
\hline FIGNL2 & MCEE & NDUFB7 & BCCIP & CCDC86 & HSPA4L \\
\hline URB1 & INTS14 & MRPS10 & PDHX & SNCA & RPL26L1 \\
\hline NBN & \begin{tabular}{|l|} 
DNAJC2 \\
\end{tabular} & NDUFAF2 & WDR36 & UBE2G1 & INTS3 \\
\hline NDUFB1 & EIF3C & WARS2 & SUPV3L1 & TOP2A & ANKZF1 \\
\hline PCCA & AKAP9 & NOP16 & EHMT2 & MRPL3 & KAT7 \\
\hline \begin{tabular}{|l|} 
POLR3D \\
\end{tabular} & MAPKAP1 & PAIP1 & GK & INTS13 & MT-ATP6 \\
\hline NF1 & NABP2 & OSBPL11 & IMPDH2 & DIEXF & WDR73 \\
\hline BRD2 & MCM7 & SFXN4 & HSPD1 & REXO4 & GEMIN4 \\
\hline NCAPD3 & NDFIP1 & BOP1 & PM20D2 & TRMT5 & STEAP3 \\
\hline SSR2 & MTA3 & TOMM40 & PDS5A & SLC25A17 & RIDA \\
\hline GTF2A2 & DCUN1D5 & ORC5 & CDKN2AIPNL & SET & TFAM \\
\hline CORO7 & THTPA & POLR1B & HSPE1 & APOO & NDUFS7 \\
\hline UBE2G2 & WRAP53 & HARS2 & POLRMT & IRF9 & RBM19 \\
\hline PPP4R3B & DPCD & FBXO22 & CLUH & TSR1 & ZNF740 \\
\hline ATP7A & HIGD2A & TIMM17A & COA7 & ALKBH5 & TTI1 \\
\hline ATR & ITPA & PABPN1 & TYMS & RARS2 & PHC2 \\
\hline MBTPS1 & FAM234A & LPCAT3 & BMS1 & INTS11 & UBE2S \\
\hline VAMP3 & TTC12 & TNPO2 & NOC3L & TBL3 & POLR1A \\
\hline PFKFB3 & CARS2 & FBL & LSAMP & ABHD11 & ORC2 \\
\hline SH3PXD2A & INCENP & SMN1,SMN2 & GNL3 & MRPS15 & \begin{tabular}{|l|} 
MRPL17 \\
\end{tabular} \\
\hline ZNF318 & ITM2C & NIP7 & SYF2 & UCK2 & MARS2 \\
\hline CWF19L1 & \begin{tabular}{|l|} 
INTS9 \\
\end{tabular} & CDK1 & STK26 & IRF2BP2 & PTCD1 \\
\hline RBX1 & POLR3B & SLC4A7 & DNPH1 & DIMT1 & DUS3L \\
\hline ULK3 & IFT57 & NDUFAF4 & NGDN & РСBP2 & CEPT1 \\
\hline TTC33 & ARMCX1 & HDDC3 & PPIF & MDC1 & PES1 \\
\hline CWC27 & TRAP1 & BRIX1 & TOMM6 & NCAPG & PWP2 \\
\hline NOL11 & DDX41 & GCA & DDX20 & HEATR1 & NT5DC2 \\
\hline KDM3B & TASOR & NCL & MGST1 & GTF2H4 & SSNA1 \\
\hline ZC3HC1 & PALD1 & EEF1E1 & TRMT10C & NUDT3 & COIL \\
\hline VRK2 & SYNPO2 & PSMF1 & PTBP3 & TGS1 & NOL10 \\
\hline DDX60 & HIGD1A & RAB11FIP5 & C1QBP & BCS1L & POLD3 \\
\hline PHACTR4 & SIK3 & TRIM65 & DNMT1 & LYAR & YY1 \\
\hline ASPM & CABIN1 & WDR43 & POLD2 & WDR3 & DCAF1 \\
\hline GPD1L & MAP3К4 & MYC & FAM162A & DDX18 & WDHD1 \\
\hline GTF3C2 & MYBBP1A & URB2 & HMGN5 & BAZ1A & MDP1 \\
\hline STAG1 & DDX21 & VRK1 & GUCY1B2 & SLC1A3 & MTMR6 \\
\hline COX6A1 & TMEM33 & UBE2D3 & RFC5 & RRP1 & NEDD4 \\
\hline SAAL1 & NPM3 & TEX10 & CDK2AP1 & POLR2F & RSL1D1 \\
\hline EDC3 & PLA2G4A & HMGA1 & DDX24 & TRRAP & SELENOO \\
\hline DTD2 & MED23 & PBX1 & UTP4 & $\mathrm{H} 1-4$ & FANCI \\
\hline SLC35E1 & PEG10 & GRPEL1 & DPH2 & DNAJA3 & HAUS1 \\
\hline CCDC115 & NOLC1 & GNL2 & POLD1 & MRPS2 & ZNF565 \\
\hline PVR & AKAP1 & PRRX1 & NDUFAF3 & PARP12 & STRIP1 \\
\hline ZCCHC3 & NELFCD & PODXL2 & PRR35 & KDM1B & BMI1 \\
\hline TRAPPC8 & SPNS1 & BLOC1S4 & UMODL1 & KIF21B & C8orf33 \\
\hline NFIX & AFP & TTN & \begin{tabular}{|l|} 
RAVER2 \\
\end{tabular} & NFATC2IP & CHD1 \\
\hline UBE2D1 & DHX38 & CDK5RAP1 & IDUA & IRAK1 & ORMDL3 \\
\hline \multicolumn{6}{|l|}{ Continued } \\
\hline
\end{tabular}


(ii) Proteins enriched more than two-fold in c-MYC-WT compared with MYCL-WT (EpiP)

\begin{tabular}{|c|c|c|c|c|c|}
\hline GPAA1 & B3GALT6 & XPC & LIN7C & VWA1 & MET \\
\hline METTL1 & COA4 & CTIF & SHPK & PNKP & \\
\hline \multicolumn{6}{|c|}{ (iii) Proteins enriched more than two-fold in c-MYC-WT compared with c-MYC- $\triangle \mathrm{MBO}$ (EpiP) } \\
\hline FAM83G & IFT20 & DOP1B & NOC3L & POLR2F & EXOC8 \\
\hline STK25 & ASPM & WIPI2 & LSAMP & KHNYN & S100A3 \\
\hline BTAF1 & GPD1L & OARD1 & ARF1 & HACD2 & VWA1 \\
\hline URB1 & STAG2 & SLC25A15 & SYF2 & RIDA & METTL1 \\
\hline PCDHGA12 & CCNYL1 & IKBKG & GXYLT1 & CEP250 & SMARCA4 \\
\hline TGFB1 & NECAP1 & MAP3K4 & NLE1 & RBM19 & PRIM2 \\
\hline SERPINE2 & PPHLN1 & UTP3 & РTBP3 & LMF2 & SHPK \\
\hline SP1 & STAG1 & CD320 & YTHDF2 & ORC2 & F8A1,F8A2,F8A3 \\
\hline GLUL & SREK1 & ARMC9 & C7orf50 & MRPL17 & BMI1 \\
\hline PHKG2 & COX6A1 & RPL36A & DNMT1 & MARS2 & C8orf33 \\
\hline NF1 & BRI3BP & LAS1L & FAM162A & PTCD1 & CCDC93 \\
\hline BRD2 & LPIN2 & PLA2G4A & HMGN5 & CEPT1 & MET \\
\hline ATP6V0C & ZNF622 & PEG10 & CDK2AP1 & LIMD2 & COA4 \\
\hline NCAPD3 & SNX21 & CCDC58 & NUP50 & ORC4 & TTC5 \\
\hline SSR2 & NUDT16 & LYRM7 & SLC16A1 & LAGE3 & CCDC63 \\
\hline PRIM1 & SAAL1 & WARS2 & ERCC4 & RMC1 & PODXL2 \\
\hline GTF2A2 & DTD2 & ZNF24 & UTP4 & NOL10 & BLOC1S4 \\
\hline EPHB3 & WASF2 & NAA16 & BUD23 & POLD3 & CDK5RAP1 \\
\hline UBE2G2 & CCDC115 & BOP1 & SELENBP1 & H2AC21 & SPATA5L1 \\
\hline PLCB3 & HAPLN3 & TNFRSF10B & SNCA & CNBP & XPC \\
\hline ATP7A & DNAJC2 & ORC5 & VPS33A & CAMLG & MPC1 \\
\hline PTK2 & AKAP9 & HARS2 & CTSC & DOCK1 & MIPEP \\
\hline HSPA14 & THTPA & TIMM17A & INTS13 & SPR & PVR \\
\hline ATR & CEP41 & PABPN1 & REXO4 & CSNK2A1 & AKAP1 \\
\hline VAMP3 & $\mathrm{XPO} 4$ & NOLC1 & PDF & CCDC51 & SRC \\
\hline PFKFB3 & CSTF2T & SMN1,SMN2 & APOO & CLPB & AFP \\
\hline DOCK11 & QRSL1 & WRNIP1 & MSH3 & YY1 & RAVER2 \\
\hline ZFYVE27 & TTC12 & HDDC3 & IGFBP5 & HEATR5A & CRLF3 \\
\hline RNASEH2B & CARS2 & STARD4 & SMYD3 & WDHD1 & DDX60 \\
\hline SH3PXD2A & \begin{tabular}{|l|} 
C17orf75 \\
\end{tabular} & GCA & RPL10L & NSD2 & HIGD1A \\
\hline TNS2 & CRLF2 & TRIM65 & INTS11 & MDP1 & EXOG \\
\hline ATG16L1 & TNFRSF12A & PBX1 & IMP3 & GLE1 & POLRMT \\
\hline TTI2 & INCENP & UQCC1 & AATF & MTMR6 & MRPS30 \\
\hline ULK3 & INTS9 & NDUFAF1 & MRPS15 & \begin{tabular}{|l|} 
THNSL1 \\
\end{tabular} & RRP1 \\
\hline TTC33 & MTRR & EHMT2 & LIG1 & RBFOX2 & CDC16 \\
\hline KDM3B & TRAP1 & HSPD1 & POLA2 & PKMYT1 & KIF21B \\
\hline TTC21B & PTBP2 & UBR5 & CCDC171 & MAP3K7 & NFATC2IP \\
\hline HEATR3 & PALD1 & CDKN2AIPNL & TGS1 & RSL1D1 & TERF2 \\
\hline ZC3HC1 & ISY1 & NAF1 & BAZ1A & FANCI & RIF1 \\
\hline VRK2 & SYNPO2 & PRORP & & & \\
\hline \multicolumn{6}{|c|}{ (iv) Commonly enriched proteins in (i) and (ii) and (iii) } \\
\hline \multicolumn{6}{|l|}{ KDM3B } \\
\hline \multicolumn{6}{|l|}{ HMGN5 } \\
\hline \multicolumn{6}{|l|}{ URB1 } \\
\hline \multicolumn{6}{|l|}{ PALD1 } \\
\hline \multicolumn{6}{|l|}{ LSAMP } \\
\hline \multicolumn{6}{|l|}{ MET } \\
\hline \multicolumn{6}{|l|}{ DNAJC2 } \\
\hline \multicolumn{6}{|l|}{ RBM19 } \\
\hline \multicolumn{6}{|l|}{ AKAP1 } \\
\hline \multicolumn{6}{|l|}{ INTS9 } \\
\hline \multicolumn{6}{|l|}{ SNCA } \\
\hline MARS2 & & & & & \\
\hline Continued & & & & & \\
\hline
\end{tabular}




\begin{tabular}{|l|l|l|l|l|l|}
\hline (iv) Commonly enriched proteins in (i) and (ii) and (iii) & & & & \\
\hline MAP3K & & & & & \\
\hline INCENP & & & & & \\
\hline AKAP9 & & & & & \\
\hline GCA & & & & & \\
\hline NCAPD3 & & & & & \\
\hline FAM162A & & & & & \\
\hline ORC5 & & & & & \\
\hline ULK3 & & & & & \\
\hline POLRMT & & & & & \\
\hline ZC3HC1 & & & & & \\
\hline PTCD1 & & & & & \\
\hline NOL10 & & & & & \\
\hline SHPK & & & & & \\
\hline XPC & & & & & \\
\hline SH3PXD2A & & & & & \\
\hline RAVER2 & & & & & \\
\hline PTBP3 & & & & & \\
\hline BOP1 & & & & & \\
\hline PEG10 & & & & & \\
\hline TIMM17A & & & & & \\
\hline SAAL1 & & & & & \\
\hline CARS2 & & & & & \\
\hline FANCI & & & & & \\
\hline HARS2 & & & & & \\
\hline BAZ1A & & & & & \\
\hline PLA2G4A & & & & & \\
\hline APOO & & & & & \\
\hline WARS2 & & & & & \\
\hline TRAP1 & & & & & \\
\hline RSL1D1 & & & & & \\
\hline
\end{tabular}

Table 2. MS analysis of identified proteins in cells reprogrammed with c-MYC. Four groups are described: (i) proteins whose peptide counts increased more than two-fold in c-MYC-WT/HDFs compared with MYCL-WT/HDFs using SeV on day 3, 5, or 7; (ii) proteins whose peptide counts increased more than two-fold in c-MYC-WT compared with MYCL-WT using EpiP; (iii) proteins whose peptide counts increased more than two-fold in c-MYC-WT compared with c-MYC- $\triangle \mathrm{MB} 0$ using EpiP; and (iv) commonly identified proteins. $n=3$ for EpiP reprogramming.

proteins were identified only in the reprogramming HDFs lysates: HNRNPK, DDX17, C1QBP, KBTBD3, COPG2, and SIKE1, of which HNRNPK, DDX17, and C1QBP are RBPs. From these results, there were 26 RBPs identified in the HDF lysates in total. We confirmed the function of the 31 proteins using a public database (https:// www.nextprot.org//) ${ }^{28}$ and found 16 of them are involved in RNA processing. A GO analysis using DAVID also showed that the 31 proteins are related to controlling pre-mRNA splicing, capping, and polyadenylation, suggesting functions in mRNA export, turnover, localization, and translation (Fig. 4E). These results suggested that MYCL interacts with RBPs via its MB2 domain and promotes reprogramming by post-transcriptional regulation.

\section{Discussion}

Here we described the molecular function of MYCL during reprogramming and compared it to the c-MYC function by focusing on MYC Box domains. We found that the MB0 and MB2 domains are important for reprogramming, and deleting either region compromised the reprogramming ability of MYCL. Proteomic analysis revealed that MYCL regulates the expression of cell adhesion-related proteins during reprogramming via the MB0 domain (Fig. 3C, D). We also found the possibility that the same domain is regulated by post-translational modifications (PTM), as discussed below. It is known that cell-substrate adhesion is closely related to the mesenchymalepithelial transition (MET) ${ }^{29}$ and that MET occurs during the reprogramming process ${ }^{30-32}$. We speculate that MYCL promotes iPSC-like colony formation via the MET process by upregulating cell adhesion-related genes. Furthermore, we identified that the MB2 domain is required for MYCL to promote reprogramming by binding to RBPs, especially RNA processing-related proteins (Fig. 4D, E). It has been reported that RBPs regulate MET through post-transcriptional regulation. For example, heterogeneous nuclear ribonucleoprotein (hnRNP) A1 regulates the alternative splicing of Rac1 to control MET ${ }^{33}$. These findings suggest that MYCL regulates the RNA processing of cell adhesion-related genes transcribed by MYCL itself or other genes. Therefore, we hypothesize 
(i) Proteins enriched more than two-fold in MYCL-WT compared with MYCL- $\triangle M B 0$ (EpiP)

\begin{tabular}{|c|c|c|c|c|c|}
\hline UBQLN2 & DYNLRB1 & EXOC2 & KRT17 & NANS & ARL1 \\
\hline REEP5 & RAB11B & ARPC2 & TPM1 & UTP15 & TMPO \\
\hline SGCD & WDR46 & CD47 & SLC44A2 & RASA1 & CETN2 \\
\hline TUBB2A & ALDH6A1 & STRN3 & FBXW10 & BAP18 & SNW1 \\
\hline HMGB1 & POTEF & PDHA1 & CAMK2D & TM9SF3 & NFS1 \\
\hline PPIC & COMMD4 & SCP2 & MT-ATP6 & YIPF5 & MPI \\
\hline HMGB3 & TUSC3 & COL3A1 & ARF4 & ATP5РB & MPDU1 \\
\hline PCNP & MYD88 & GNB2 & NUCKS1 & LIMS1 & USP15 \\
\hline SYAP1 & RAC1 & COG6 & TMED2 & LRBA & OVCA2 \\
\hline CHMP4B & MACF1 & SOX2 & PRKACA & MAPK14 & CALD1 \\
\hline UTP3 & SRSF11 & SSR1 & MID1 & DUSP12 & GBP1 \\
\hline BLOC1S1 & EXOC5 & CSTF3 & PCID2 & THOC3 & ANP32B \\
\hline ST6GALNAC1 & DNAJC9 & CACNA2D1 & TRIP12 & SRSF5 & USP48 \\
\hline MAP3K20 & MICAL1 & NRDC & GADD45GIP1 & VAC14 & PDIA4 \\
\hline KNTC1 & POLR2L & MSRB3 & GTF2I & PDXDC1 & SF3A3 \\
\hline BAG5 & SFXN3 & NOL11 & ERLIN2 & ZNF462 & NUCB2 \\
\hline CD320 & CRIP1 & OSTC & DBNL & ITGB1 & AGK \\
\hline MRPL11 & NAA10 & RPL37A & STT3A & CTTN & PPIL3 \\
\hline TFG & ARF6 & LZIC & PAFAH1B1 & EEF1B2 & IDI1 \\
\hline THYN1 & CCDC43 & BMP1 & SLC25A24 & UGP2 & ELN \\
\hline NDRG3 & NEK7 & PDCL3 & HOOK3 & LSM2 & COL4A2 \\
\hline TMX4 & \begin{tabular}{|l|} 
TUBB4B \\
\end{tabular} & UGP2 & LAS1L & ACTB & BUD31 \\
\hline SEPHS1 & MYO1E & TNS3 & HLA-H & RABL3 & MAP2 \\
\hline RPL36A & FNTA & VPS26B & DCN & RWDD1 & PAIP1 \\
\hline MYDGF & SRBD1 & EHD1 & PUM1 & TOMM20 & ITGA5 \\
\hline OPTN & DBI & ANKFY1 & PUS7 & CRABP2 & GPX8 \\
\hline GNAQ & SUGP2 & KBTBD3 & KPNA4 & VDAC3 & TXLNG \\
\hline ACSL4 & MTA1 & SCPEP1 & METTL26 & EDIL3 & ATP5MG \\
\hline ATP5ME & MAP7D1 & FBLN2 & B4GALT4 & PLA2G4A & PIK3R4 \\
\hline ABI3BP & ACTG1 & LDLR & MBD5 & CTNNA1 & PLBD2 \\
\hline ASAH1 & HINT1 & EXOSC7 & CSRP1 & RPL23A & ZYX \\
\hline GNS & HMGN1 & DIP2B & GNB1 & TMEM165 & COX7C \\
\hline WDR61 & PTGR1 & PITRM1 & \begin{tabular}{|l|} 
SNRPB2 \\
\end{tabular} & DNAH6 & H3-3A,H3-3B \\
\hline ARL8A & TMSB4X & METTL14 & CNN2 & DPP9 & NCKAP1 \\
\hline MAP3K20 & FAM114A1 & TMSB10 & PPIB & ENDOD1 & AHNAK \\
\hline NDRG1 & FTH1 & CNPY3 & S100A10 & NDUFB11 & PGM2 \\
\hline PITPNA & SGTA & HABP2 & C1orf198 & NAA50 & PODXL \\
\hline NIF3L1 & SGPL1 & SRP9 & MARCKSL1 & DNAJC8 & CFL2 \\
\hline NME2 & CD59 & NDUFB9 & TOR1AIP1 & NXN & STAT6 \\
\hline PFDN1 & DHRS4 & RBPJ & NDUFA4 & MRPS17 & TP53BP1 \\
\hline ATG3 & GSPT1 & DCTN5 & ACSS2 & REXO2 & ATAD1 \\
\hline ACIN1 & BLOC1S3 & TMED1 & GSTK1 & PEBP1 & EIF3K \\
\hline RAB14 & RFC3 & AKR1B1 & ISLR & S100A13 & GPX7 \\
\hline SNAP23 & CD55 & TALDO1 & NOP14 & SLC25A6 & TRIO \\
\hline EMC2 & RPS15A & DSTN & POLR2A & OSBPL3 & TSPYL5 \\
\hline \multicolumn{6}{|c|}{ (ii) Proteins enriched more than wo-fold in c-MYC- $\triangle M B 0$ compared with MYCL- $\triangle M B 0$ (EpiP) } \\
\hline UBQLN2 & ACIN1 & NEK7 & PITRM1 & PDLIM5 & CAMK2D \\
\hline HMGB1 & SRSF11 & RAB11B & ITGA1 & H3-3A,H3-3B & CRABP2 \\
\hline REEP5 & OPTN & ANKFY1 & ISLR & Clorf198 & NCKAP1 \\
\hline NDRG1 & KNTC1 & CD47 & SRSF5 & LUZP1 & HMGN1 \\
\hline SGCD & PAIP1 & PRKACA & ERLIN2 & IDI1 & MAP2 \\
\hline MAP3K20 & NIF3L1 & SCRN3 & MRPS17 & HABP2 & TOR1AIP1 \\
\hline TUBB2A & POTEF & FAM114A1 & TOMM20 & PAFAH1B1 & ARL1 \\
\hline CCDC43 & COG6 & NOLC1 & UGP2 & ITGA5 & OSBPL3 \\
\hline PCNP & TSPYL5 & COMMD4 & LSM2 & CHIC1 & ANP32B \\
\hline TMX4 & DYNLRB1 & EHD1 & UTP15 & SMTN & STT3A \\
\hline
\end{tabular}


(ii) Proteins enriched more than wo-fold in c-MYC- $\triangle$ MB0 compared with MYCL- $\triangle M B O$ (EpiP)

\begin{tabular}{|c|c|c|c|c|c|}
\hline BLOC1S1 & STRN3 & PPP4C & PUM1 & DUSP12 & VPS26B \\
\hline SYAP1 & ASAH1 & FNTA & MYO1E & MEAF6 & APP \\
\hline PPIC & ATP5ME & GNAQ & TP53RK & NMT1 & GALNT1 \\
\hline NME2 & OVCA2 & SEMA7A & POLR2L & NDUFA4 & SSR1 \\
\hline ACSL4 & RAC1 & RPL36A & MYO6 & LIMS1 & YIF1A \\
\hline HMGB3 & OSTC & FTH1 & PUS7 & CLASP2 & UGP2 \\
\hline FBXW10 & DBI & PTGR1 & PIK3R4 & ILF3 & USP48 \\
\hline MAP3K20 & PDHA1 & KRT17 & MYD88 & RASA1 & PPIB \\
\hline NDRG3 & SFXN3 & ARPC2 & REXO2 & GALE & METTL26 \\
\hline GNS & TNS3 & ABI1 & DBNL & TXLNG & MYO1D \\
\hline UTP3 & DHRS4 & PAIP1 & USP15 & PPIL3 & \begin{tabular}{|l|} 
NDUFS6 \\
\end{tabular} \\
\hline SEPHS1 & MPDU1 & PCID2 & SRBD1 & RFC3 & PPIF \\
\hline BAG5 & \begin{tabular}{|l|} 
DNAJC9 \\
\end{tabular} & SEC24A & ACTG1 & SCLY & SMARCC2 \\
\hline ATG3 & MAPK14 & UBQLN1 & LZIC & MPV17 & STAM2 \\
\hline SCPEP1 & MRPS24 & MTA1 & TRIP12 & VAC14 & BMP1 \\
\hline TFG & PLA2G4A & BAP18 & THOC3 & DCTN5 & PGM2 \\
\hline MT-ATP6 & NOL11 & METTL14 & SCP2 & COG7 & CERS2 \\
\hline MRPL11 & SUGP2 & EXOC5 & \begin{tabular}{|l|} 
POLR2A \\
\end{tabular} & MCRIP1 & SLIT3 \\
\hline CHMP4B & TUSC3 & BLOC1S3 & CSTF3 & CTNNA1 & HOOK3 \\
\hline SGPL1 & DIP2B & DUSP23 & GNB2 & SENP3 & COL3A1 \\
\hline MICAL1 & PFDN1 & SOX2 & GSPT1 & EIF2B1 & ITGB1 \\
\hline DCN & NRDC & LDLR & GADD45GIP1 & SETD7 & AGK \\
\hline CUL4A & SGTA & CNPY3 & KPNA4 & DNASE2 & MPHOSPH10 \\
\hline ARF6 & PODXL & MPI & FADS2 & RAI14 & BOLA2,BOLA2B \\
\hline HLA-H & ARL8A & HINT1 & NSA2 & TPMT & IMPACT \\
\hline MACF1 & PDXDC1 & GSTK1 & ATAD1 & EDIL3 & USP9X \\
\hline THYN1 & CD320 & CD59 & METAP1 & IGF2R & OCIAD2 \\
\hline MYDGF & PITPNA & FBLN2 & TPM1 & NAA50 & CACNA2D1 \\
\hline NACA4P & MAP7D1 & NANS & MBD5 & EEF1B2 & YIPF5 \\
\hline NAA10 & WDR46 & \begin{tabular}{|l|} 
TM9SF3 \\
\end{tabular} & DCTD & EEA1 & TMSB10 \\
\hline NDUFB9 & LRRC17 & YAP1 & GTF2I & CD55 & SNAP23 \\
\hline ABI3BP & WDR61 & RABL3 & PRKRA & DNAJC8 & EIF3H \\
\hline SNW1 & LMCD1 & KLF4 & PSMD4 & S100A10 & KBTBD3 \\
\hline SNRPB2 & COL4A2 & CFL2 & LSM5 & FBXO22 & ACYP1 \\
\hline ARF4 & VDAC3 & ALDH6A1 & CYP51A1 & CBX3 & SNX27 \\
\hline ATP5MG & CNN1 & CLINT1 & ACSS2 & EIF2B3 & SLC25A24 \\
\hline CTTN & CALD1 & DYNC1LI1 & NDUFAF2 & S100A13 & CDC42BPB \\
\hline TMPO & EWSR1 & ANTXR2 & TP53BP1 & RBM17 & IDH3G \\
\hline RPL37A & SPATS2L & PHLDB1 & CARHSP1 & CFAP74 & EMC2 \\
\hline PLS1 & HSD17B7 & FAP & NXN & TUBB4B & SF3A3 \\
\hline MTR & OSTF1 & TMSB4X & SRP9 & VASP & EXOC2 \\
\hline NCBP1 & SFXN4 & NUCKS1 & RPS29 & MANF & COPS6 \\
\hline VPS4B & AHNAK & CSRP1 & \begin{tabular}{|l|} 
SLC25A6 \\
\end{tabular} & CARM1 & FKBP2 \\
\hline TMOD3 & RBPJ & \begin{tabular}{|l|} 
TALDO1 \\
\end{tabular} & ATP5PB & PRPF4B & TBC1D15 \\
\hline GPX7 & B4GALT4 & MSRB3 & DNAH6 & COX7C & OGFR \\
\hline ALDH1L2 & CRIP1 & AP3M1 & SCAMP2 & C1orf50 & ELN \\
\hline TSN & TOR1B & PDS5A & PDIA4 & RWDD1 & GABPA \\
\hline DYNLL1 & USP47 & ENDOD1 & NUCB2 & GINS4 & DR1 \\
\hline FKBP5 & OAS2 & MRTO4 & NFS1 & NPC2 & $\mathrm{ABI} 2$ \\
\hline SERPINE2 & NDUFAF4 & ZYX & HACD3 & EXOSC7 & \\
\hline \multicolumn{6}{|c|}{ (iii) Commonly enriched proteins in (i) and (ii) } \\
\hline WDR46 & TUSC3 & RBPJ & NFS1 & NIF3L1 & DNAJC8 \\
\hline MYO1E & MYD88 & FBXW10 & ARF4 & TM9SF3 & TMPO \\
\hline RAC1 & SFXN3 & CAMK2D & MPDU1 & CTNNA1 & EEF1B2 \\
\hline TMSB10 & MACF1 & NUCKS1 & OVCA2 & CHMP4B & COL3A1 \\
\hline LDLR & DBNL & PRKACA & ANP32B & PCNP & S100A10 \\
\hline Continued & & & & & \\
\hline
\end{tabular}




\begin{tabular}{|c|c|c|c|c|c|}
\hline \multicolumn{6}{|c|}{ (iii) Commonly enriched proteins in (i) and (ii) } \\
\hline CRABP2 & EXOC5 & TRIP12 & SLC25A24 & PAIP1 & NEK7 \\
\hline NOL11 & DNAJC9 & GADD45GIP1 & SF3A3 & ARL1 & MICAL1 \\
\hline POLR2L & CRIP1 & GTF2I & AGK & PODXL & S100A13 \\
\hline TOMM20 & CCDC43 & AHNAK & PPIL3 & REXO2 & STT3A \\
\hline CFL2 & SRBD1 & ENDOD1 & IDI1 & FNTA & H3-3A,H3-3B \\
\hline POTEF & SUGP2 & PDHA1 & ELN & FBLN2 & SCPEP1 \\
\hline TSPYL5 & MTA1 & HOOK3 & COL4A2 & NDRG3 & TXLNG \\
\hline SGTA & TMSB4X & HLA-H & MAP2 & EXOC2 & MPI \\
\hline REEP5 & ACTG1 & PUM1 & ATP5MG & SOX2 & HMGN1 \\
\hline SGCD & HINT1 & KPNA4 & PIK3R4 & DBI & UBQLN2 \\
\hline HMGB3 & PTGR1 & METTL26 & COX7C & TUBB2A & NDUFB9 \\
\hline SYAP1 & EMC2 & B4GALT4 & NCKAP1 & SRP9 & PDXDC1 \\
\hline UTP3 & RPL36A & MBD5 & ASAH1 & FAM114A1 & TOR1AIP1 \\
\hline BLOC1S1 & CD59 & SNRPB2 & PGM2 & CD55 & MAP7D1 \\
\hline MAP3K20 & NUCB2 & C1orf198 & TP53BP1 & SNAP23 & COG6 \\
\hline KNTC1 & BLOC1S3 & ACSS2 & ATAD1 & UGP2 & TNS3 \\
\hline BAG5 & STRN3 & GSTK1 & RPL37A & KRT17 & SGPL1 \\
\hline CD320 & SCP2 & NANS & RFC3 & DCTN5 & CSRP1 \\
\hline TFG & GNB2 & UTP15 & TALDO1 & USP48 & ATG3 \\
\hline THYN1 & SSR1 & BAP18 & ISLR & NDUFA4 & EDIL3 \\
\hline TMX4 & CSTF3 & YIPF5 & \begin{tabular}{|l|} 
POLR2A \\
\end{tabular} & ITGB1 & ZYX \\
\hline ARL8A & CACNA2D1 & LIMS1 & OSBPL3 & АТР5РВ & PCID2 \\
\hline GNAQ & NRDC & THOC3 & GPX7 & PUS7 & RASA1 \\
\hline ACSL4 & MSRB3 & SRSF5 & PPIC & SRSF11 & DNAH6 \\
\hline ATP5ME & OSTC & VAC14 & MYDGF & USP15 & ALDH6A1 \\
\hline ABI3BP & LZIC & ARF6 & HMGB1 & MT-ATP6 & COMMD4 \\
\hline GNS & BMP1 & UGP2 & ERLIN2 & NAA10 & CNPY3 \\
\hline MAP3K20 & VPS26B & LSM2 & FTH1 & ITGA5 & HABP2 \\
\hline NDRG1 & ANKFY1 & RABL3 & DUSP12 & CD47 & DHRS4 \\
\hline NME2 & KBTBD3 & RWDD1 & CTTN & MRPL11 & SNW1 \\
\hline PFDN1 & EHD1 & PLA2G4A & PPIB & OPTN & ARPC2 \\
\hline MAPK14 & EXOSC7 & SLC25A6 & SEPHS1 & WDR61 & TPM1 \\
\hline ACIN1 & DIP2B & NAA50 & DCN & PDIA4 & GSPT1 \\
\hline DYNLRB1 & PITRM1 & NXN & PAFAH1B1 & VDAC3 & TUBB4B \\
\hline RAB11B & METTL14 & MRPS17 & PITPNA & CALD1 & \\
\hline
\end{tabular}

Table 3. MS analysis of identified proteins in cells reprogrammed with MYCL-WT and c-MYC- $\Delta$ MB0 compared with MYCL- $\triangle \mathrm{MB}$. Three groups are described: (i) proteins whose peptide counts increased more than two-fold in MYCL-WT compared to MYCL- $\triangle \mathrm{MB0}$; (ii) proteins whose peptide counts increased more than two-fold in c-MYC- $\triangle \mathrm{MB} 0$ compared to MYCL- $\triangle \mathrm{MB} 0$; and (iii) commonly identified proteins. $n=3$.

that transcriptional and post-transcriptional regulation by MYCL promotes MET, which increases the efficiency of reprogramming and leads to higher quality iPSCs.

Western blotting revealed that MYCL protein has a unique expression pattern (Supplementary Fig. S8 and $\mathrm{S} 12$ ). The calculated molecular weight of MYCL is about $40 \mathrm{kDa}$ (364 aa), but we detected three strong bands at around $60 \mathrm{kDa}$, which we verified with second antibody (Supplementary Fig. S13). Since the expression of MYCL- $\triangle$ MB0 showed a strong single band, we speculate that the MYCL MB0 domain is the PTM site (Supplementary Fig. S8). Such a phenomenon was not observed in c-MYC (Supplementary Fig. S7). One possible type of relevant PTM is phosphorylation. Phosphorylation is crucial for protein function. For example, RNA polymerase II ( $\mathrm{Pol}$ II) is required for transcription pauses in a promoter-proximal position during transcription initiation. In order to initiate transcription, the C-terminal domain of Pol II must be phosphorylated by P-TEFb ${ }^{34}$. In addition, the phosphorylation of c-MYC on threonine 58 in the MB1 domain promotes c-MYC binding to F-box and WD repeat domain containing 7 (FBXW7), causing the ubiquitination of c-MYC, which triggers c-MYC degradation ${ }^{35}$. Similarly, MYCL might undergo phosphorylation to change its activity and interaction with binding proteins. However, this hypothesis requires further study.

Comprehensive proteomic analysis suggested that the MYCL MB0 domain influences the expression of cell adhesion-related proteins, and MYCL shows an up-regulation of phosphorylated cytoskeletal proteins (Fig. 3C, D, and Supplementary Fig. S11A). Cell adhesion is mediated by adhesion molecules, such as integrins and cadherins, which function in the extracellular matrix (ECM) and cell-cell adhesion and are important for cell 
A

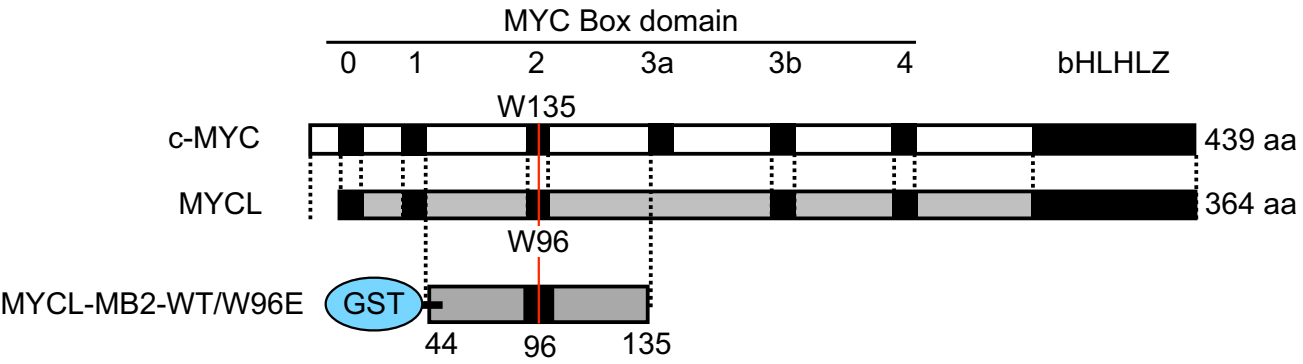

B

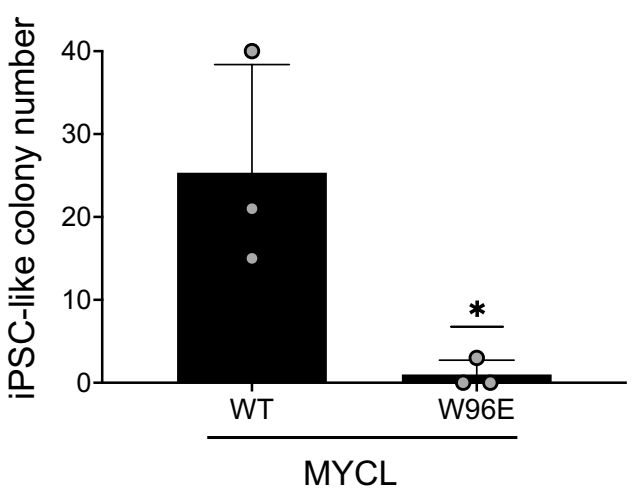

C

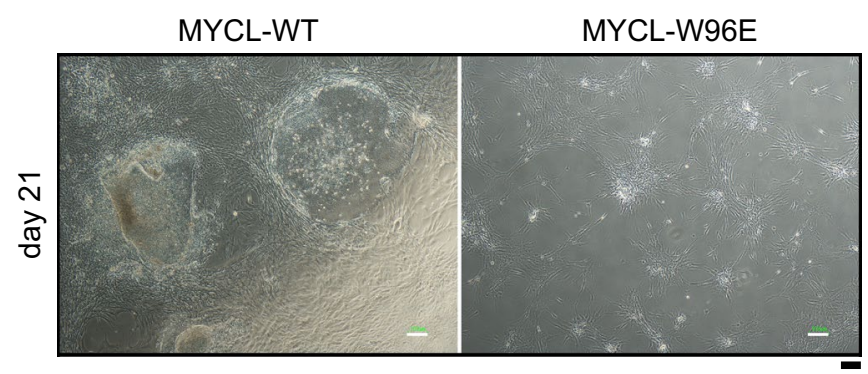

E

GO analysis

Molecular function

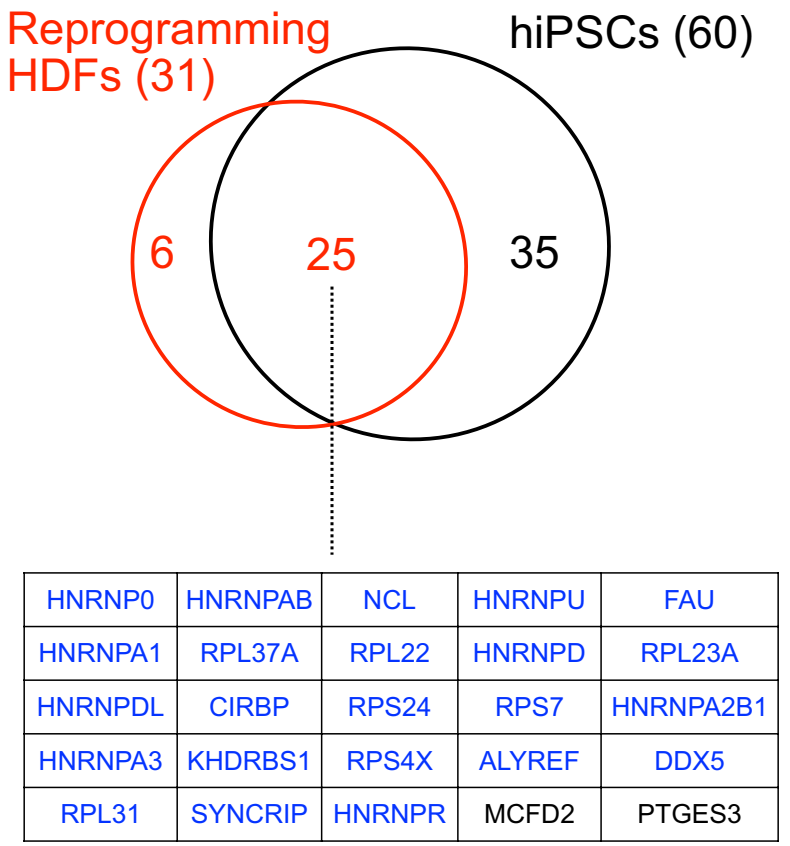

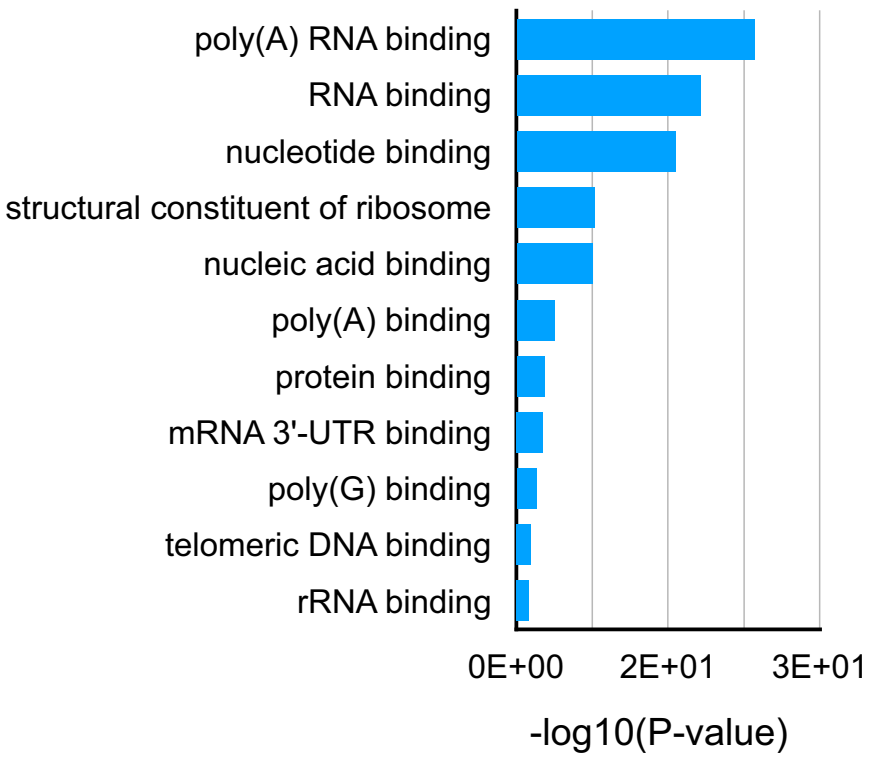

Figure 4. MYCL regulates RNA processing-related proteins during reprogramming via the MB2 domain. (A) W96 and W135 in the MB2 domain of MYCL and c-MYC, respectively. The structure with the recombinant protein of the MB2 domain of MYCL-WT/W96E is shown below. The numbers on the right indicate amino acid lengths. (B) The number of iPSC-like and non-iPSC-like colonies derived from $1 \times 10^{5} \mathrm{HDF}$ transduced with EpiP including MYCL-WT or MYCL-W96E on day 21 . Mean \pm SD values are shown. $n=3,{ }^{*} p<0.05$ by unpaired $t$-test. (C) Representative images of reprogramming HDFs 21 days after the transduction of EpiP, including MYCL-WT or MYCL-W96E. Scale bars, $100 \mu \mathrm{m}$. (D) Venn diagram of enriched proteins between reprogramming HDFs and hiPSCs by AP-MS. A list of the 25 commonly enriched proteins is shown below. Blue indicates RBP (23 in total). (E) Molecular function from the GO analysis of the 25 commonly identified proteins in (D). 


\begin{tabular}{|c|c|c|c|c|c|}
\hline \multicolumn{6}{|c|}{ (i) Proteins enriched more than two-fold in MYCL-MB2-WT compared with MYCL-MB2-W96E (reprogramming HDFs) (31) } \\
\hline HNRNPR & HNRNPK & DDX17 & C1QBP & $F A U$ & RPL22 \\
\hline RPS7 & CIRBP & MCFD2 & KBTBD3 & COPG2 & PTGES3 \\
\hline \multicolumn{6}{|l|}{ SIKE1 } \\
\hline \multicolumn{6}{|c|}{ (ii) Proteins enriched more than two-fold in MYCL-MB2-WT compared with MYCL-MB2-W96E (hiPSCs) (60) } \\
\hline HNRNPA1 & HNRNPD & HNRNPDL & HNRNPA2B1 & HNRNPAO & HNRNPA3 \\
\hline KHDRBS1 & HNRNPAB & ALYREF & DDX5 & HNRNPU & SYNCRIP \\
\hline HNRNPR & HNRNPK & PRPF31 & DDX42 & LYAR & YBX1 \\
\hline$F A U$ & RPL22 & RPL37A & RPL23A & $N C L$ & RPS24 \\
\hline PTGES3 & MCFD2 & CFDP1 & ACTR2 & ITGB1 & SFRP1 \\
\hline \multirow[t]{3}{*}{ DSTN } & PDHB & HADHA & HADHB & NDUFS5 & CNBP \\
\hline & Italic value & RNA binding proteins & & & \\
\hline & Bold italic value & : RNA processing proteins & & & \\
\hline \multicolumn{6}{|c|}{ (iii) Common enriched proteins enriched between reprogramming HDFs and hiPSCs (25) } \\
\hline HNRNPA1 & HNRNPD & HNRNPDL & HNRNPA2B1 & HNRNPAO & HNRNPA3 \\
\hline KHDRBS1 & HNRNPAB & ALYREF & DDX5 & HNRNPU & SYNCRIP \\
\hline HNRNPR & $F A U$ & RPL37A & RPL22 & RPL23A & CIRBP \\
\hline RPS24 & RPS7 & RPS4X & RPL31 & $N C L$ & PTGES3 \\
\hline \multicolumn{6}{|l|}{ MCFD2 } \\
\hline
\end{tabular}

Table 4. AP-MS analysis of identified proteins in MYCL-MB2-WT using cell lysates from reprogrammed HDFs and hiPSCs. Three groups are described: (i) protein interactors whose peptide counts increased in reprogramming HDFs more than two-fold in MYCL-MB2-WT than MYCL-MB2-W96E; (ii) protein interactors whose peptide counts increased in hiPSCs more than two-fold in MYCL-MB2-WT compared to MYCL-MB2W96E; and (iii) commonly identified proteins. $n=1$.

communication and the regulation of fundamental physiological processes such as tissue development and maintenance ${ }^{36,37}$. Human iPSCs and hESCs have unique focal adhesion localization, and appropriate adhesion to the ECM is required to regulate reprogramming via MET and maintain pluripotency ${ }^{38-40}$. Accordingly, our study supports MYCL regulating cell-substrate adhesion through its MB0 domain to promote reprogramming. In other words, MYCL might regulate proteins involved in cell adhesion and the cytoskeleton directly or indirectly to cause MET and promote reprogramming. In c-MYC, loss of the MB0 domain positively affects iPSC-like colony formation, suggesting that this domain has a different function compared to MYCL. This functional difference is somewhat surprising since the domain is well conserved (Supplementary Fig. S5B). We would like to clarify this point in the future.

We also found that the MB2 domain has an important function in MYCL-reprogramming (Fig. 2B,C). Deleting the MB2 domain completely compromised the reprogramming ability of MYCL. In c-MYC, the MB2 domain has an important function in transformation activity ${ }^{14}$, and tryptophan 135 in the MB2 domain is essential for this activity. The equivalent tryptophan residue in MYCL is tryptophan 96. MYCL has little transformation activity, but we showed that the mutation of tryptophan 96 completely lost the reprogramming ability of MYCL. To further investigate the function, we sought interacting proteins by affinity column chromatography. We found 31 proteins, including 26 RBPs, that interact with the MYCL MB2 domain (Table 4, genes written in blue). A GO analysis suggested that some of the 31 proteins are involved in RNA processing (Table 4). It has been reported that altered RNA processing affects somatic cell reprogramming ${ }^{41}$. Therefore, we hypothesize that MYCL also promotes MET in reprogramming by regulating RNA processing via interactions with RBPs at its MB2 domain. An illustrative summary of how MYCL regulates cell reprogramming through these two domains is shown in Fig. 5.

To conclude, we have demonstrated that MYCL promotes more efficient reprogramming than c-MYC, regulates the expression of cell adhesion and cytoskeletal proteins, and is involved in RNA processing via a single tryptophan residue in the MB2 domain. Following these findings, we propose that MYCL causes MET by regulating the expression of proteins involved in the promotion of reprogramming from the RNA-processing stage. 
Further elucidation of the function of MYCL in reprogramming will improve the quality and efficiency of iPSC generation.

\section{Material and methods}

Cell culture. HDFs (106-05f.) were purchased from Cell Applications, Inc. HDFs were cultured in DMEM (08459-64, Nacalai Tesque) supplemented with 10\% FBS (10439-024, gibco) and 1\% penicillin and streptomycin (15140-122, Pen/Strep, gibco). The hiPSC clone 201B7 was used in this study ${ }^{2}$. iPSCs were cultivated on iMatrix-511 (NP892-012, Nippi)-coated $\left(0.5 \mu \mathrm{g} / \mathrm{cm}^{2}\right)$ cell culture plates with StemFit (AK03N, Ajinomoto) supplemented with bFGF and passaged via dissociation into single cells using TrypLE Select (A12859-01, Life Technologies) on day 7 following a previously reported protocol ${ }^{42}$.

Generation of iPSCs. A frozen stock of HDFs was thawed and cultured for four days, and then $1 \times 10^{5}$ cells were collected by trypsinization. With SeV, HDFs were transduced with the CytoTune-iPS 2.0 (c-MYC) or CytoTune-iPS 2.0L (MYCL) Sendai Reprogramming Kit (DV-0304, DV-0305, ID Pharma). With EpiP, HDFs were electroporated with $1.2 \mu \mathrm{g}$ of plasmid mixtures with the Neon Transfection System (MPK1096 and MPK10096, Invitrogen). The plasmid mixtures included pCXLE-SOX2, -KLF4, -OCT3/4-shp53, -LIN28A, and pCXWBEBNA1 with wild-type or mutant pCXLE-c-MYC or -MYCL ${ }^{17}$. The mixing ratio of SOX2, KLF4, OCT3/4-shp53, LIN28A, EBNA1, and c-MYC/MYCL was 1:1:2:1:0.5:2. After that, the cells were plated in a 6-well plate and cultured in StemFit AK03N without bFGF with iMatrix-511 at $0.25 \mu \mathrm{g} / \mathrm{cm}^{2}$ in SeV or $0.125 \mu \mathrm{g} / \mathrm{cm}^{2}$ in EpiP. The culture medium was changed the next day and every three days after that. The colonies were counted 21 days after plating.

Episomal plasmid vector construction for deletion mutants of c-MYC and MYCL. We previously generated pCXLE-c-MYC and -MYCL from human cDNAs encoding c-MYC and MYCL amplified by PCR and cloned into pENTR1 $\mathrm{A}^{17}$. Primers for the deletion mutants were designed using the Primer Design tool for the In-Fusion HD Cloning Kit (639650, Takara) and inserted into pENTR1A. The switch from pENTR1A to pCXLE was done using the Gateway system (11791020, Invitrogen). The primers used are listed in Table S1.

Immunostaining. The cells were fixed with 4\% formaldehyde (163-20145, Wako) for $20 \mathrm{~min}$ at room temperature. Then, the fixed cells were treated with PBS (14249-24, Nacalai Tesque) containing $0.5 \%$ Triton X-100 (35501-15, Nacalai Tesque) and 3\% bovine serum albumin (01281-84, BSA, Nacalai Tesque) for $20 \mathrm{~min}$ at room temperature for permeabilization. The cells were incubated with primary antibodies diluted in PBS containing $3 \% \mathrm{BSA}$ at $4^{\circ} \mathrm{C}$ overnight. After washing with PBS, the cells were incubated with fluorescence-conjugated secondary antibodies for $1 \mathrm{~h}$ at room temperature. Nuclei were visualized with Hoechst 33342 (346-07951, DOJINDO). Anti-TRA-1-60 (1:500, 560071, BD Pharmingen, and 1:500, 09-0068, Stemgent) and Alexa 488-conjugated goat anti-mouse $\operatorname{IgG}, \operatorname{IgM}(\mathrm{H}+\mathrm{L})(1: 250, \mathrm{~A} 10680$, Invitrogen) were used as the antibodies.

Imaging and quantification. Stained cells were imaged using a BZ-9000 imaging system (KEYENCE) or ArrayScan High-Content Systems (Thermo Fisher Scientific). HCS Studio 2.0 Cell Analysis Software (Thermo Fisher Scientific) was used to quantify cell counts and signal intensities. The Cellomics BioApplication system (Thermo Fisher Scientific) was programmed to capture and analyze 25 images per well. The total cell number was detected by Hoechst 33342 staining. The number of TRA-1-60 $(+)$ cells was calculated as the number of TRA-1-60 (+) cells among Hoechst $(+)$ cells. TRA-1-60 (+) cells were calculated by dividing this number by the total cell number.

Flow cytometry. Transduced cells were harvested with $0.25 \%$ trypsin/1 mM EDTA (25200-056, gibco) each day after the transduction for the analysis. At least $5 \times 10^{4}$ cells were stained with the following antibodies in FACS buffer (2\% FBS, 0.36\% glucose (16806-25, Nacalai Tesque), $50 \mu \mathrm{g} / \mu \mathrm{L}$ Pen/Strep in PBS) for $30 \mathrm{~min}$ at room temperature: BV510-conjugated anti-TRA-1-60 (1:40, 563188, BD Biosciences) and PE-Cy7-conjugated anti-CD13 (1:40, 561599, BD Biosciences) antibodies. The analysis was performed using MACSQuant Analyzers (Miltenyi Biotec). Negative controls used a mixture of HDFs without any EpiP transduction and reprogramming HDFs electroporated with EpiP including c-MYC or MYCL. "Isotype" means mixed HDFs stained with the isotype control of anti-TRA-1-60 (1:40, 563082, BD Biosciences) and -CD13 (1:40, 557646, BD Biosciences) antibodies.

SDS-PAGE. Cells were lysed with SDS sample buffer (0.125 M Tris-base (35434-21, Nacalai Tesque), $0.96 \mathrm{M}$ glycine (17109-35, Nacalai Tesque), and 17.3 mM SDS (31606-75, Nacalai Tesque)) containing 3-mercaptoethanol (139-16452, Wako). Samples were applied and separated in an 8\% polyacrylamide gel composed of $30 \%$ (w/v)-Acrylamide/Bis Mixed Solution (29:1) (06141-35, Nacalai Tesque), Separating Gel Buffer Solution (4x) (30651-05, Nacalai Tesque) and Stacking Gel Buffer Solution (4x) (32158-25, Nacalai Tesque) for SDS-PAGE.

Western blotting. Proteins on an SDS-PAGE gel were transferred to a PVDF membrane (IPVH00010, Immobilon-P, Millipore) and probed with the following antibodies using an iBind Flex system (SLF2000, SLF2010 and SLF2020, Invitrogen): anti-human MYCL (1:250, AF4050, R\&D) (1:250, C-20, sc-790, Santa Cruz), anti-human c-MYC (1:500, 9E10, sc-40, Santa Cruz, and 1:500, D84C12, CST), anti- $\beta$-actin (1:1000, A5441, SIGMA), anti-Goat (1:3000, ab6741-1, abcam), anti-mouse (1:3000, 7076S, CST), and anti-rabbit (1:3000, 7074S, CST) antibodies. 


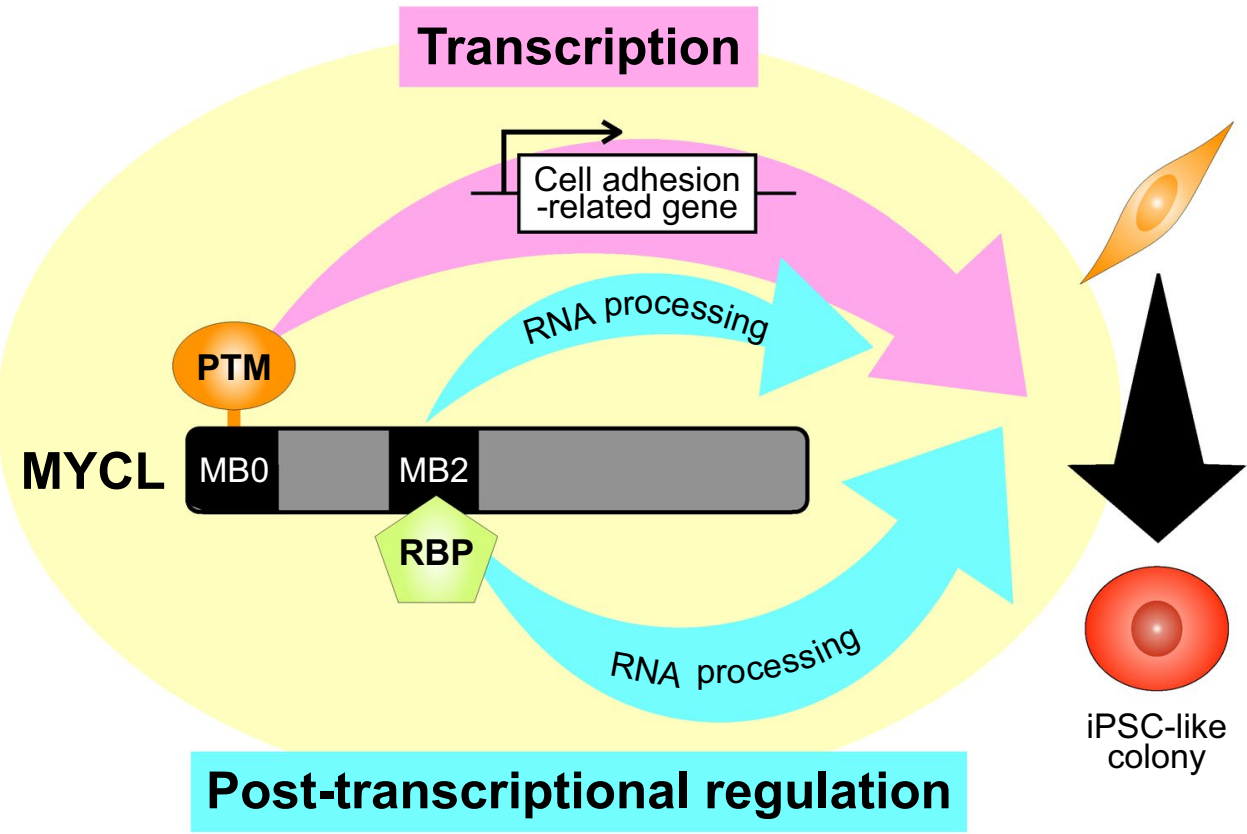

Figure 5. Model of the reprogramming process by MYCL. MYCL promotes iPSC-like colonies via its MB0 and MB2 domains. The MB0 domain regulates the expression of cell-adhesion proteins, possibly via posttranslational modifications (PTM). The MB2 domain regulates RNA processing by interacting with RNAbinding proteins (RBP). We speculate that MYCL promotes reprogramming through the synergistic effects of these two mechanisms.

Preparation of recombinant proteins and affinity purification (AP). The MB2 region of MYCLWT or -W96E was cloned into pGEX-6P-1. The plasmids were transformed into BL21 E. coli (DE3) (L1198, Promega) competent cells. The fusion proteins, GST-MYCL-WT-MB2 and GST-MYCL-W96E-MB2, were induced by treatment with $0.5 \mathrm{mM}$ IPTG (19742-94, Nacalai Tesque) for $4 \mathrm{~h}$ at $37^{\circ} \mathrm{C}$. The proteins were purified using glutathione Sepharose beads (17-0756-01, GE Healthcare). Human iPSCs or reprogramming HDFs were lysed in RIPA buffer (20 mM Tris/HCl (pH 7.6) (35436-01, Nacalai Tesque), 1\% NP-40 (25223-75, Nacalai Tesque), $0.1 \%$ SDS, $150 \mathrm{mM} \mathrm{NaCl}$ (31320-05, Nacalai Tesque), and protease inhibitor (25955-11, Nacalai Tesque)) and then centrifuged. Cell lysates (supernatant) were transferred into a column (29922, Thermo Fisher Scientific) packed with beads conjugated with GST- MYCL-WT or -W96E proteins. After washing, binding proteins were eluted in lysis buffer (12 mM sodium deoxycholate (190-08313, Wako), $12 \mathrm{mM}$ sodium lauroyl sarcosinate (19210382, Wako), and $100 \mathrm{mM}$ Tris-HCl (pH9.0) (314-90381, NIPPON GENE)) for the MS analysis. The iPSC lysates were prepared 6 days after passaging in two $10-\mathrm{cm}$ dishes $(n=1)$, and reprogramming HDF lysates were prepared 3 days after $\mathrm{SeV}$ transduction in five 10 -cm dishes $(n=1)$.

GO analysis by DAVID. The Database for Annotation, Visualization, and Integrated Discovery (DAVID Bioinformatics Resources 6.8) was used to identify enriched biological GO terms and KEGG pathway ${ }^{43-45}$. For more information, please visit the DAVID website (https://david.ncifcrf.gov/home.jsp) and KEGG Database website (https://www.kegg.jp/kegg/kegg1.html).

The methods for MS are described in the Supplementary methods.

Received: 16 August 2021; Accepted: 1 December 2021

Published online: 20 December 2021

\section{References}

1. Takahashi, K. \& Yamanaka, S. Induction of pluripotent stem cells from mouse embryonic and adult fibroblast cultures by defined factors. Cell 126, 663-676 (2006).

2. Takahashi, K. et al. Induction of pluripotent stem cells from adult human fibroblasts by defined factors. Cell 131, 861-872 (2007).

3. Azuma, K. \& Yamanaka, S. Recent policies that support clinical application of induced pluripotent stem cell-based regenerative therapies. Regen. Ther. 4, 36-47 (2016).

4. Huang, C.-Y. et al. Human iPSC banking: barriers and opportunities. J. Biomed. Sci. 26, 87 (2019).

5. Moradi, S. et al. Research and therapy with induced pluripotent stem cells (iPSCs): social, legal, and ethical considerations. Stem Cell Res. Ther. 10, 341 (2019).

6. Koyanagi-Aoi, M. et al. Differentiation-defective phenotypes revealed by large-scale analyses of human pluripotent stem cells. Proc. Natl. Acad. Sci. 110, 20569-20574 (2013).

7. Nakagawa, M., Takizawa, N., Narita, M., Ichisaka, T. \& Yamanaka, S. Promotion of direct reprogramming by transformationdeficient Myc. Proc. Natl. Acad. Sci. 107, 14152-14157 (2010). 
8. Baluapuri, A., Wolf, E. \& Eilers, M. Target gene-independent functions of MYC oncoproteins. Nat. Rev. Mol. Cell Biol. 21, 255-267 (2020).

9. Varmus, H. E. The molecular genetics of cellular oncogenes. Annu. Rev. Genet. 18, 553-612 (1984).

10. Oster, S. K., Mao, D. Y. L., Kennedy, J. \& Penn, L. Z. Functional analysis of the N-terminal domain of the Myc oncoprotein. Oncogene 22, 1998-2010 (2003).

11. Beaulieu, M.-E., Castillo, F. \& Soucek, L. Structural and biophysical insights into the function of the intrinsically disordered myc oncoprotein. Cells 9, 1038 (2020).

12. Amati, B. et al. Transcriptional activation by the human c-Myc oncoprotein in yeast requires interaction with Max. Nature 359, 423-426 (1992).

13. Zhang, Q. et al. MB0 and MBI are independent and distinct transactivation domains in MYC that are essential for transformation. Genes 8, 134 (2017).

14. Kalkat, M. et al. MYC protein interactome profiling reveals functionally distinct regions that cooperate to drive tumorigenesis. Mol. Cell 72, 836-848.e7 (2018).

15. Fusaki, N., Ban, H., Nishiyama, A., Saeki, K. \& Hasegawa, M. Efficient induction of transgene-free human pluripotent stem cells using a vector based on Sendai virus, an RNA virus that does not integrate into the host genome. Proc. Jpn. Acad. Ser. B 85, 348-362 (2009).

16. Tanabe, K., Nakamura, M., Narita, M., Takahashi, K. \& Yamanaka, S. Maturation, not initiation, is the major roadblock during reprogramming toward pluripotency from human fibroblasts. Proc. Natl. Acad. Sci. 110, 12172-12179 (2013).

17. Okita, K. et al. A more efficient method to generate integration-free human iPS cells. Nat. Methods 8, 409-412 (2011).

18. Xing, Q. R. et al. Diversification of reprogramming trajectories revealed by parallel single-cell transcriptome and chromatin accessibility sequencing. Sci. Adv. 6, eaba1190 (2020)

19. Wolf, E. \& Eilers, M. Targeting MYC proteins for tumor therapy. Annu. Rev. Cancer Biol. 4, 61-75 (2020).

20. Schwanhäusser, B. et al. Global quantification of mammalian gene expression control. Nature 473, 337-342 (2011).

21. Huang, D. W., Sherman, B. T. \& Lempicki, R. A. Systematic and integrative analysis of large gene lists using DAVID bioinformatics resources. Nat. Protoc. 4, 44-57 (2009).

22. Huang, D. W., Sherman, B. T. \& Lempicki, R. A. Bioinformatics enrichment tools: paths toward the comprehensive functional analysis of large gene lists. Nucl. Acids Res. 37, 1-13 (2009).

23. Wasylishen, A. R. et al. New model systems provide insights into Myc-induced transformation. Oncogene 30, 3727-3734 (2011).

24. Okita, K., Ichisaka, T. \& Yamanaka, S. Generation of germline-competent induced pluripotent stem cells. Nature 448, 313-317 (2007).

25. Yu, J. et al. Induced pluripotent stem cell lines derived from human somatic cells. Science 318, 1917-1920 (2007).

26. Sone, M. et al. Hybrid cellular metabolism coordinated by Zic3 and Esrrb synergistically enhances induction of naive pluripotency. Cell Metab. 25, 1103-1117.e6 (2017).

27. Maekawa, M. et al. Direct reprogramming of somatic cells is promoted by maternal transcription factor Glis1. Nature 474, 225-229 (2011).

28. Zahn-Zabal, M. et al. The neXtProt knowledgebase in 2020: data, tools and usability improvements. Nucl. Acids Res. 48, D328-D334 (2020).

29. Rodriguez-Boulan, E. \& Macara, I. G. Organization and execution of the epithelial polarity programme. Nat. Rev. Mol. Cell Biol. 15, 225-242 (2014).

30. Li, R. et al. A mesenchymal-to-epithelial transition initiates and is required for the nuclear reprogramming of mouse fibroblasts. Cell Stem Cell 7, 51-63 (2010).

31. Sakurai, K. et al. Kinome-wide functional analysis highlights the role of cytoskeletal remodeling in somatic cell reprogramming. Cell Stem Cell 14, 523-534 (2014).

32. Pei, D., Shu, X., Gassama-Diagne, A. \& Thiery, J. P. Mesenchymal-epithelial transition in development and reprogramming. Nat. Cell Biol. 21, 44-53 (2019).

33. Bonomi, S. et al. HnRNP A1 controls a splicing regulatory circuit promoting mesenchymal-to-epithelial transition. Nucl. Acids Res. 41, 8665-8679 (2013).

34. Price, D. H. Regulation of RNA polymerase II elongation by c-Myc. Cell 141, 399-400 (2010).

35. Welcker, M. et al. The Fbw7 tumor suppressor regulates glycogen synthase kinase 3 phosphorylation-dependent c-Myc protein degradation. Proc. Natl. Acad. Sci. 101, 9085-9090 (2004).

36. Tamkun, J. W. et al. Structure of integrin, a glycoprotein involved in the transmembrane linkage between fibronectin and actin. Cell 46, 271-282 (1986).

37. Hynes, R. Integrins: A family of cell surface receptors. Cell 48, 549-554 (1987).

38. Närvä, E. et al. A strong contractile actin fence and large adhesions direct human pluripotent colony morphology and adhesion. Stem Cell Rep. 9, 67-76 (2017).

39. Santoro, R., Perrucci, G. L., Gowran, A. \& Pompilio, G. Unchain My heart: integrins at the basis of iPSC cardiomyocyte differentiation. Stem Cells Int. 2019, 1-20 (2019).

40. Hansson, J. et al. Highly coordinated proteome dynamics during reprogramming of somatic cells to pluripotency. Cell Rep. 2, 1579-1592 (2012)

41. Ohta, S., Nishida, E., Yamanaka, S. \& Yamamoto, T. Global splicing pattern reversion during somatic cell reprogramming. Cell Rep. 5, 357-366 (2013).

42. Nakagawa, M. et al. A novel efficient feeder-free culture system for the derivation of human induced pluripotent stem cells. Sci. Rep. 4, 3594 (2014).

43. Kanehisa, M. \& Goto, S. KEGG: Kyoto encyclopedia of genes and genomes. Nucleic Acids Res. 28, 27-30 (2000).

44. Kanehisa, M. Toward understanding the origin and evolution of cellular organisms. Protein Sci. 28, 1947-1951 (2019).

45. Kanehisa, M., Furumichi, M., Sato, Y., Ishiguro-Watanabe, M. \& Tanabe, M. KEGG: integrating viruses and cellular organisms. Nucl. Acids Res. 49, D545-D551 (2021).

\section{Acknowledgements}

This research was supported by AMED under Grant Number JP21bm0104001 and a grant from the Fujiwara Memorial Incorporated Foundation. We thank Dr. C. Okubo, Dr. H. Kagawa, Dr. T. Yamakawa, Dr. K. Okita, and Dr. K. Takahashi for scientific discussions; Dr. A. Ohta and Dr. Y. Nishi for technical assistance with the ArrayScan analysis; and Dr. P. Karagiannis for reading the manuscript.

\section{Author contributions}

C.A. and M.N. wrote the main manuscript text. C.A. mainly prepared all figures (supported by C.S., Y.C., T.I., and M.N.). Proteome analysis was done by Y.K. and M.I.. All authors reviewed the manuscript. 


\section{Competing interests}

M.I. is a scientific adviser (without salary) of xFOREST therapeutics. Other authors do not provide a competing interest statement.

\section{Additional information}

Supplementary Information The online version contains supplementary material available at https://doi.org/ 10.1038/s41598-021-03260-5.

Correspondence and requests for materials should be addressed to M.N.

Reprints and permissions information is available at www.nature.com/reprints.

Publisher's note Springer Nature remains neutral with regard to jurisdictional claims in published maps and institutional affiliations.

(c) Open Access This article is licensed under a Creative Commons Attribution 4.0 International License, which permits use, sharing, adaptation, distribution and reproduction in any medium or format, as long as you give appropriate credit to the original author(s) and the source, provide a link to the Creative Commons licence, and indicate if changes were made. The images or other third party material in this article are included in the article's Creative Commons licence, unless indicated otherwise in a credit line to the material. If material is not included in the article's Creative Commons licence and your intended use is not permitted by statutory regulation or exceeds the permitted use, you will need to obtain permission directly from the copyright holder. To view a copy of this licence, visit http://creativecommons.org/licenses/by/4.0/.

(c) The Author(s) 2021 